\title{
Microwave spectro-polarimetry of matter and radiation across space and time
}

\section{Jacques Delabrouille, et al. [full author details at the end of the article]}

Received: 29 July 2020 / Accepted: 2 March 2021 / Published online: 3 July 2021

(C) The Author(s) 2021

\begin{abstract}
This paper discusses the science case for a sensitive spectro-polarimetric survey of the microwave sky. Such a survey would provide a tomographic and dynamic census of the three-dimensional distribution of hot gas, velocity flows, early metals, dust, and mass distribution in the entire Hubble volume, exploit CMB temperature and polarisation anisotropies down to fundamental limits, and track energy injection and absorption into the radiation background across cosmic times by measuring spectral distortions of the CMB blackbody emission. In addition to its exceptional capability for cosmology and fundamental physics, such a survey would provide an unprecedented view of microwave emissions at sub-arcminute to few-arcminute angular resolution in hundreds of frequency channels, a data set that would be of immense legacy value for many branches of astrophysics. We propose that this survey be carried out with a large space mission featuring a broad-band polarised imager and a moderate resolution spectro-imager at the focus of a $3.5 \mathrm{~m}$ aperture telescope actively cooled to about $8 \mathrm{~K}$, complemented with absolutely-calibrated Fourier Transform Spectrometer modules observing at degree-scale angular resolution in the 10-2000 GHz frequency range. We propose two observing modes: a survey mode to map the entire sky as well as a few selected wide fields, and an observatory mode for deeper observations of regions of specific interest.
\end{abstract}

Keywords Cosmology · Early Universe · Galaxies · Galaxy clusters

For endorsement and a broader overview of all the related microwave spectro-polarimetric Voyage 2050 White Papers please visit here: www.microwave-spectropolarimetry.eu

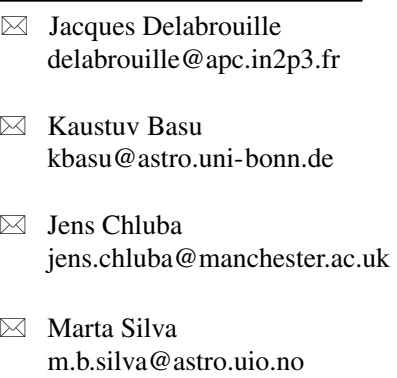




\section{Introduction}

In the past few decades, a standard model of cosmology, $\Lambda \mathrm{CDM}$, has emerged. According to our understanding, our Universe is about 13.8 billion years old, and expanded from an initial hot and dense state. Baryonic matter accounts for $5 \%$ of its present matter-energy content, non-baryonic dark matter for about $25 \%$, and some unknown form of energy with negative pressure, dubbed dark energy, for the remaining $70 \%$. The galaxies and clusters of galaxies that we observe today form by the gravitational collapse of tiny primordial fluctuations of the spacetime metric, thought to originate from an early stage of fast accelerated expansion, known as cosmic inflation.

But very fundamental questions remain unanswered: we do not know what the dark matter and dark energy are, whether dark matter interacts, or if extra light particles exist. We are unsure whether inflation did indeed take place and exactly what physics was at work in the very early Universe. We still have to reconcile the laws of gravitation with the standard model of particle interactions-both of which are known to be incomplete and require extensions to explain existing observations. We do not know the topology of the Universe, or whether it is finite or infinite. We do not fully understand how structure forms, or why some structures on small scales appear to be incompatible with $\Lambda \mathrm{CDM}$ predictions. We do not have a convincing explanation for anomalies in the large-scale statistics of cosmic microwave background (CMB) anisotropies, except invoking chance multipole alignments and excursions in tails of the realization of a Gaussian random field.

The distribution of matter and energy in the Universe, by virtue of being the product of physical laws and their effect on the Universe's constituents, encodes answers to these questions. We thus propose to conduct an unprecedented full census of this distribution, over scales from one arcminute to the entire sky, and over $99 \%$ of cosmic history. The census will be carried out using a high-angular-resolution, highsensitivity spectro-polarimetric survey of the microwave sky, which will track faint signatures of matter and radiation interactions across cosmic time, exploit the CMB as a multi-faceted cosmology probe, and construct a three-dimensional picture of the various components of the cosmic web, across space and time, using five main observables:

1. tracers of the interaction of the $\mathrm{CMB}$ with free electrons in the cosmic web (Sunyaev-Zeldovich effects), to map the distribution of hot gas, its temperature, and large-scale velocity flows;

2. CMB deflections by gravitational lensing, used as a tracer of mass in the entire Hubble volume;

3. high-redshift dust and line emission, to map atoms in structures across cosmic time;

4. primary $\mathrm{CMB}$ anisotropies at the cosmic-variance limit, to constrain parameters of $\Lambda \mathrm{CDM}$ and its extensions or alternatives;

5. distortions of the $\mathrm{CMB}$ blackbody spectrum, to probe the thermal history of the Universe and all processes that can impact it up to redshifts of a few million. 
None of these can be observed with high accuracy in full isolation from the others. Most of them probe interconnected phenomena, which justifies combining the observations. The combined survey will provide a comprehensive and detailed view of the history of the Universe, and a tomographic and dynamic census of the threedimensional distribution of hot gas, velocity flows, early metals, dust, and mass distribution. In addition to its exceptional capability for cosmology, this survey will be extremely valuable for many other branches of astrophysics.

The proposed survey requires an L-class space mission, featuring three instruments that observe within the $10-2000 \mathrm{GHz}$ frequency range, at varying spectral and angular resolutions. Two of these instruments will be located at the focus of a large (3-m class) cold ( $\sim 8 \mathrm{~K}$ ) telescope, providing arcminute-scale angular resolution at $300 \mathrm{GHz}$. A broad-band, multifrequency, polarimetric imager will provide sensitive observations of the CMB and of Sunyaev-Zeldovich (SZ) effects, while a moderate spectral resolution $(R \simeq 300)$ filter-bank spectrometer will map the infrared (IR) background and atomic and molecular lines out to high redshift. These instruments will comprise tens of thousands of mm and sub-mm detectors, which will be cooled to sub-kelvin temperatures for sky background-limited performance.

An additional guest focal plane instrument, compatible with the overall constraints of the mission, can be considered for increased science outcomes beyond the mission's primary science goals. ${ }^{1}$

On the same platform (or on an independent spacecraft) absolute spectroscopy across the entire frequency range will be performed by a Fourier-transform spectrometer (FTS) consisting of one or a few independent FTS modules, covering the full 10-2000 GHz band with spectral resolution ranging from 2.5 to $60 \mathrm{GHz}$, angular resolution ranging from a fraction of a degree to a few degrees, and overall sensitivity $<1 \mathrm{Jy} \mathrm{sr}^{-1}, 4$ to 5 orders of magnitude better than that of COBE-FIRAS.

We envision 6 years of observation from an orbit around the L2 Sun-Earth point, with two different observing modes: a survey for about half the mission time, to map the entire sky as well as a few selected wide fields; and an observatory mode, during which the rest of the time will be made available to the wider scientific community for an opportunity to observe regions of specific interest.

Although ambitious, the proposed survey builds upon previous space mission concepts already studied at the pre-phase-A or phase-A levels (CORE, PICO, and $S P I C A)$. The FTS instrument builds on similar technology flown on $C O B E$ and Herschel, and on the previously proposed PIXIE and PRISTINE mission designs.

Past experience teaches us that progress in cosmology has often come in unexpected ways through opening up new directions. By systematically probing the Universe using many approaches, and with an unprecedented capability to observe faint signals coming from the largest cosmological distances, the proposed mission will be key in pushing back the frontiers of our understanding of the Universe that we live in. It will be transformational in many areas of physics, astrophysics, and cosmology at the most fundamental level, in a way that is unmatched by any other existing or proposed experiment—and can only be achieved from space.

\footnotetext{
${ }^{1}$ For instance, an instrument such as the proposed BBOP instrument, originally designed for SPICA, could extend the polarization observations to higher frequencies [1]
} 
While one cannot guarantee completely resolving all the open questions we plan to address, the survey proposed is guaranteed to transform our knowledge of the Universe. It will also be of immense legacy value for many branches of astrophysics, with an unprecedented view of microwave emissions at sub-arcminute to few-arcminute angular resolution in hundreds of frequency channels.

\section{Scientific introduction}

\subsection{Open questions in cosmology}

The twentieth century witnessed the spectacular transformation of physical cosmology into a quantitative branch of science and has ushered in the era of "precision cosmology". During the past two decades a standard cosmological model has emerged: inflationary $\Lambda$ CDM. Seven independent parameters describe the matter and energy content of the Universe, its expansion history, and the statistical distribution of initial perturbations that evolve to form the large-scale structures we observe today [2].

Even though $\Lambda \mathrm{CDM}$ provides a good phenomenological fit to most cosmological observations, it lacks an underlying theoretical explanation, and is, at best, strikingly incomplete. In the absence of observations capable of discriminating between different options, parameters in natural extensions to $\Lambda \mathrm{CDM}$ are set to default values driven by simplicity. For the minimal $\Lambda \mathrm{CDM}$, we do not know the nature of $95 \%$ of the contents of the Cosmos. Inflation explains a host of phenomena including the origin of density perturbations and the topology of the Universe, but we do not have a physical model for it.

Not all current observations match the theoretical expectations of $\Lambda \mathrm{CDM}$. For the lowest multipoles, the amplitude of CMB fluctuations are somewhat too low, enough to motivate investigations for possible explanations. Hemispherical power asymmetry and alignments of CMB multipoles on large scales question the assumptions of homogeneity and statistical isotropy. There is a significant $4 \sigma$ tension in the determination of the Hubble constant between CMB and distance-ladder measurements [3], and there are apparent inconsistencies with observations of structures at galaxy and cluster scales [4].

These inconsistencies may disappear with refinements of theoretical modeling and data analyses, or may point to new physics, as do the existence of dark matter and dark energy, inflation, and the incompleteness of particle physics. Are we missing an essential piece of the puzzle? Current data are not sufficient to provide definitive answers.

The matter-energy constituents of the Universe, the laws of gravitation and particle interactions, and the initial state of metric perturbations, all impact the distribution of matter structures and radiation in the Universe. By mapping the components of our Universe across space and time at the next level of detail, we will open a new window on the properties of the Universe, and on the machinery that governs cosmic evolution and encodes the fundamental laws of nature. 


\subsection{Cosmological observations in the microwave sky}

Confidence in inflationary $\Lambda \mathrm{CDM}$ relies on its spectacular consistency with observations of the CMB anisotropy, and with other cosmological probes (Ref. [5], and references therein). The primary $\mathrm{CMB}^{2}$ gives an image of structures and velocity flows in a thin shell at $z \simeq 1100$, and hence also at a specific time. Cosmic variance ${ }^{3}$ precludes stringent statistical tests of the global cosmological paradigm on the largest scales using primary CMB data alone.

The CMB is a bath of radiation that permeates the entire Universe and interacts with everything in it. Every process that exchanges energy with the cosmic microwave photons leaves an imprint. We propose to exploit these imprints to extract answers to the key open questions in astrophysics by making measurements at microwave and submillimeter wavelengths of four sets of related phenomena:

1. interactions of the $\mathrm{CMB}$ photons with structures of matter through gravitational lensing and through scattering with electrons. Observations of these "secondary CMB anistropies" will probe structures throughout most of the Hubble volume (Section 3);

2. microwave emission of baryonic matter (cosmic dust, atoms, and molecules) residing in the cosmic web out to high redshift. Mapping this emission and combining it with maps of secondary CMB anisotropies will give a tomographic view of the Universe on large scales (Section 4);

3. primary $\mathrm{CMB}$ temperature and polarization anisotropies. Higher fidelity measurements will give new insights on the cosmological model and on the fundamental laws of physics (Section 5);

4. distortions of the CMB blackbody spectrum. Searching for distortions will open a new window to investigate phenomena predicted by minimal $\Lambda \mathrm{CDM}$ or its extensions, and will provide unique discovery space for unexpected phenomena (Section 6).

These observables are linked because none of them can be observed in isolation. All contribute to the total emission observed in the microwave band, and each is a source of confusion when measuring others. None of these signals can be fully exploited without understanding them all - and many of them provide answers to closely related cosmological questions.

\section{A census of structures with the $\mathrm{CMB}$ as a backlight}

Current experiments have vividly demonstrated the enormous potential of using the $\mathrm{CMB}$ as a backlight to study the cosmic matter distribution and its evolution. A

\footnotetext{
2 'Primary anisotropy' refers to patterns arising from processes at the last scattering surface. 'Secondary' refers to processes the $\mathrm{CMB}$ photons undergo between the last scattering surface and our telescopes.

${ }^{3}$ 'Cosmic variance' represents variance in measurements arising from the limited statistics of observing a single Universe.
} 
high-sensitivity, full-sky survey with arcminute angular resolution and frequency coverage spanning the millimeter and submillimeter wavebands would map the distribution of essentially all baryonic and dark-matter structures in the observable Universe, and measure the peculiar motion of matter within the cosmic web. Such a complete matter census would be transformational.

After leaving the surface of last scattering, CMB photons interact with intervening matter primarily via two processes: (1) scattering by electrons in ionized plasma, called the Sunyaev-Zeldovich (SZ) effect; and (2) deflection by gravitational potential wells along the cosmic web.

There are several variants of the SZ effect. The thermal SZ (tSZ) traces the integral of gas pressure in the baryons associated with large-scale structures along the line of sight. Galaxy clusters are the most dominant contributors. The magnitude of the spectral difference of the tSZ relative to the CMB is called "the Compton- $y$ " parameter. The kinetic SZ (kSZ) traces line-of-sight gas momentum with respect to the CMB. Relativistic effects (rSZ) subtly alter the tSZ frequency spectrum and can be used to directly measure gas temperatures (e.g., without relying on X-ray data). There are additionally non-thermal SZ (ntSZ) signals that can constrain the particle composition of exotic plasmas, such as radio bubbles driven by AGN feedback, and polarized SZ (pSZ) probes of cluster transverse motions and internal substructure.

Gravitational potentials lens the CMB backlight [6-8]. By cross-correlating CMB lensing maps with visible tracers, such as galaxies and clusters, we can partition the lensing signal into redshift slices, a process known as "lensing tomography," and measure the growth of structure back to redshifts of a few, well past the point where galaxy cosmic shear becomes ineffective due to lack of background sources. On smaller scales, the lensing effect probes deflections by strong localized overdensities, enabling determination of cluster masses out to redshifts beyond the reach of galaxy shear measurements $(z>2)$ [9]; this technique will be essential for using high- $z$ clusters as a cosmological tool. CMB lensing can also be used to detect cluster's transverse motions through the moving-lens effect [10,11], complementing kinetic and polarised SZ measurements of the cosmic velocity field.

The CMB provides an ideal backlight for these studies because: (1) it originates from a known redshift; (2) its spectrum at emission is known to be an almost perfect blackbody; and (3) its statistical properties are well defined. It is a new and powerful tool for a comprehensive census of matter in the Universe.

\subsection{Hot gas in the cosmic web}

Within only a decade, the number of galaxy clusters detected via the tSZ effect has increased from four to well over two thousand [12-15]. A sensitive space mission with close to $1^{\prime}$ resolution would find all galaxy groups and clusters throughout the observable Universe with masses above $5 \times 10^{13} \mathrm{M}_{\odot}$, achieving a complete census of these objects from the moment of their appearance. The resulting catalog would yield the ultimate cosmological constraints from cluster counts and clustering, and deliver a goldmine for astrophysical studies of clusters, spawning countless follow-up observations. 
Beyond massive bound objects, the SZ effects open new avenues of research into unbound gas residing in the filaments of the cosmic web in the form of the warmhot intergalactic medium (IGM), and the circumgalactic medium (CGM) trapped within galaxy dark-matter halos. $90 \%$ or more of all baryonic mass resides in the IGM and CGM. However, at their characteristic temperatures and densities, these phases defy other means of detection, hiding the majority of baryons under poorly understood conditions. The SZ effects are an effective tool for mapping these cosmic constituents. A high-precision all-sky map of the Compton- $y$ parameter traces the thermal energy in these phases, while the kSZ effect outlines their spatial density distribution. Combining them, we can extract key information on the physical state of the IGM and CGM, establishing a whole new class of constraints on viable feedback mechanisms [16]. More generally, such measurements will finally close our census of the state of all observable matter in the Universe. These goals cannot be achieved by any other means.

Removal of astrophysical foregrounds is important at the anticipated signal levels. For example, far-IR thermal emission from dust within galaxies severely contaminates the tSZ signal in lower mass systems (see Fig. 1) because the tSZ signal roughly scales as $M^{1.6}$, whereas dust emission is roughly $\propto M$. Observations with multiple frequencies at sub-millimeter wavelengths (from $300 \mathrm{GHz}$ to $1 \mathrm{THz}$ ) is essential, something that can only be realistically achieved from space.

Wide spectral coverage is also central to the other SZ revolution in the coming decade, namely "SZ spectroscopy," i.e., accurately measuring the SZ spectrum to separate its various components: the rSZ, kSZ, and ntSZ effects (and potentially pSZ too, as well as faint signals from multiple-scattering SZ effects). Data from Planck has already motivated studies along these lines [17-19].

\subsection{Large-scale velocity flows}

The kinematic SZ effect gives the motion of objects with respect to the CMB rest frame [20]. The cosmic velocity field is a well-known cosmological probe (of dark energy, for example), complementary to measures of the density field. Furthermore,
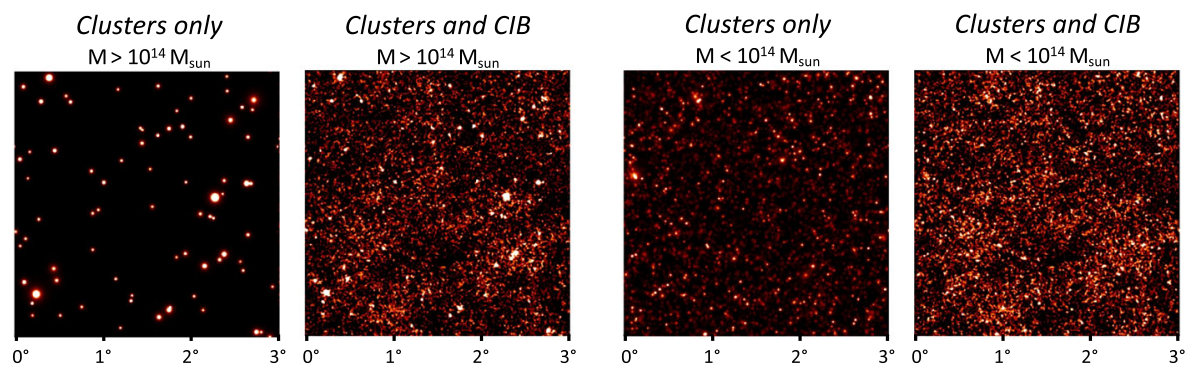

Fig. 1 Impact of the Cosmic Infrared Background (CIB) for detecting low-mass galaxy clusters at $150 \mathrm{GHz}$. Left two panels: simulated clusters of more than $10{ }^{14} \mathrm{M}_{\odot}$, without and with $150-\mathrm{GHz}$ CIB fluctuations added. Right two panels: same but with clusters of mass between $1 \times 10^{13}$ and $10^{14} \mathrm{M}_{\odot}$. All figures use the same color scale 
combining the two makes it possible to test for possible deviations from general relativity on scales that only cosmology can access.

The kSZ signal is sensitive to the line-of-sight component of the bulk proper motions of ionised gas, and scales with the free-electron optical depth, irrespective of the thermal energy. For clusters and groups of galaxies, the kSZ signal is of the order 1-10 $\mu \mathrm{K}$, just beyond the reach of current-generation CMB experiments for detection in individual objects. However, cross-correlation with other tracers, particularly galaxy surveys, has produced statistical detections [21, 22]. The scope of kSZ applications will dramatically expand in the coming decade with wide sky coverage and increasing sensitivity. This will improve leverage for various cross-correlation studies and also enable individual detections to break the "optical depth degeneracy" currently limiting kSZ applications for cosmology. Together with a smaller contribution from the moving-lens effect via lensing (of the order $0.01-0.1 \mu \mathrm{K}$ ), mapping the complete $3 \mathrm{D}$ velocity flows in the Universe will be possible.

It is important to note that the spectrum of the $\mathrm{kSZ}$ emission law is identical to that of the CMB (ignoring small higher-order effects). Hence, kSZ signals are best measured on arcminute angular scale in CMB intensity maps free from contamination of foregrounds (including tSZ and the $\mathrm{CIB}$ ), and with low primary CMB fluctuations. This underscores the need for high-precision component separation on small scales, which can only be achieved with a large number of frequency channels spanning frequencies that are only observable from space.

\subsection{Survey requirements for mapping hot gas and velocity flows}

The observation of ionized gas in clusters and filaments that constitute the cosmic web requires the detection of hot gas using the spectral signature of the tSZ effect, and discrimination of the various contributions (i.e., thermal, relativistic, kinematic, and non-thermal effects).

Sensitivity and angular resolution The left panel of Fig. 2 shows the modeled distribution of clusters of different masses as a function of $Y_{500}$ (integral of the $y$-parameter out to a radius $r_{500}$, the radius inside of which the average density is 500 times the critical density). To detect (at more than $5 \sigma$ ) all clusters above $5 \times 10^{13} \mathrm{M}_{\odot}$, we need a sensitivity of $\delta Y_{500}=9 \times 10^{-7}$ or better, with the goal of trying to go down to $\delta Y_{500}=5 \times 10^{-8}$, to detect groups of $10^{13} \mathrm{M}_{\odot}$.

The right panel of Fig. 2 shows that all clusters of mass above $5 \times 10^{13} \mathrm{M}_{\odot}$ have angular diameter larger than $\simeq 0.8^{\prime}$. We require an angular resolution on the reconstructed $y$ map of $\simeq 1.5^{\prime}$. Because of the extended cluster profile, this yields only a maximum sensitivity loss of about $20 \%$ by reason of beam dilution (for detecting an isolated cluster). To reduce blending effects, we target a goal of $1^{\prime}$ angular resolution.

A cluster with mass $M>5 \times 10^{13} \mathrm{M}_{\odot}$ has typical Compton optical depth $\tau \simeq$ $10^{-3}$. For a peculiar velocity of $300 \mathrm{~km} \mathrm{~s}^{-1}$, the $\mathrm{kSZ}$ amplitude is about $3 \mu \mathrm{K}$. Detecting this at $5 \sigma$ for a cluster of $1^{\prime}$ angular size requires a CMB sensitivity of $0.6 \mu \mathrm{K}$ arcmin (goal). A $3 \sigma$ detection can be achieved with a CMB sensitivity of $1 \mu \mathrm{K}$ arcmin. Measuring $\mathrm{kSZ}$ on large clusters is challenging because of confusion with the primary CMB. However, the $\mathrm{kSZ}$ effect dominates over the primary CMB at 

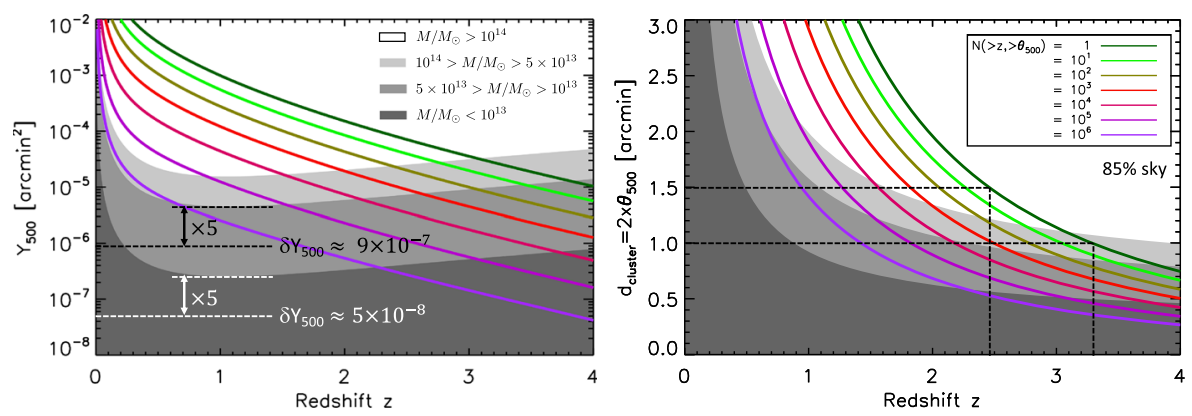

Fig. 2 Left: Distribution of clusters for various mass ranges as a function of redshift and cluster-integrated Compton parameter $Y_{500}$ (white and gray areas of various shades), modeled following the self-similar model of [23]. A survey with tSZ flux error $\delta Y_{500} \simeq 9 \times 10^{-7}$ would detect all clusters of mass $M>$ $5 \times 10^{13} \mathrm{M}_{\odot}$ (about 1.5 million objects), while $\delta Y_{500} \simeq 5 \times 10^{-8}$ would be sufficient to even detect groups of $\simeq 10^{13} \mathrm{M}_{\odot}$. Colored lines show, as a function of $z$ and $Y_{500}$, the expected number of clusters that have both larger tSZ signal, and are located at higher redshift. Right: Distribution of clusters as a function of redshift and angular size, with the same white and gray color code. All clusters of mass $M>10^{14} \mathrm{M}_{\odot}$ (white) have an angular size larger than $\simeq 1^{\prime}$, and clusters $>5 \times 10^{13} \mathrm{M}_{\odot}$ (white and light gray) larger than $\simeq 0.8^{\prime}$. Colored lines show, as a function of $z$ and cluster angular diameter $d_{\text {cluster }}$, the expected number of clusters that have both larger angular diameter, and are located at higher $z$. Dashed lines show that the highest redshift clusters with angular sizes $1.5^{\prime}$ are at $z \simeq 2.5$, and with size $1^{\prime}$ at $z \simeq 3.3$

$\ell>4000$, i.e., angular scales smaller than $3^{\prime}$. Hence, there is a preference for better angular resolution (requirement $1.5^{\prime}$, goal $1^{\prime}$ ), to measure kSZ for clusters smaller than the primary CMB damping angular scale and hence to avoid much of the extra CMB noise (see left panel of Fig. 3).

Frequency range and number of channels The survey must allow one to separate the tSZ and kSZ signals of interest from other astrophysical sources of emission, in particular the small-scale thermal dust and radio emission from extragalactic sources, either those associated with the cluster, or those that constitute the overall radio and infrared source background and contribute to the overall sky noise.

In fields away from Galactic contamination, using at least 3 frequency channels to detect and characterize radio-sources, at least 3 for IR sources, and around 6 for the various SZ effects and the primary CMB with some redundancy, leads to at least a dozen frequency channels being required for separating the various components when all of those are above the instrumental noise (as is likely to be the case for many of the interesting clusters detected by a sensitive survey). Over a large fraction of sky, extra channels are needed also to isolate small-scale emission from the Galactic interstellar medium (i.e., synchrotron and free-free, plus thermal and anomalous dust emission), for a total of around 20 frequency bands. These channels should cover the frequency range where the SZ signals are the strongest relative to other emissions (between 100 and $400 \mathrm{GHz}$ ), with channels around the tSZ minimum, null, and maximum $(150,220$, and $350 \mathrm{GHz})$, and extra channels at lower and higher frequencies to characterize low- and high-frequency foregrounds.

As seen in Fig. 1, fluctuations of the CIB are a serious source of noise for detecting clusters below $10^{14} \mathrm{M}_{\odot}$, even at $150 \mathrm{GHz}$; however, if the CIB can be reduced 
to $\simeq 20 \%$ of its initial amplitude, tSZ emission from clusters below $10^{14} \mathrm{M}_{\odot}$ become detectable. This should be feasible over most of the sky if the survey includes observations between $300 \mathrm{GHz}$ and $800 \mathrm{GHz}$, where CIB fluctuations dominate on small scales. Such observations must be done from space.

CIB fluctuations are still more problematic for measuring the fainter rSZ correction (typically less than $10 \%$ of the $\mathrm{tSZ}$, and seen at higher frequency). A wide spectroscopic survey of the CIB in the 300-1000 GHz frequency range, allowing for decomposition into contributions from different redshifts and/or components, will help characterize and subtract this contaminant to the best possible accuracy.

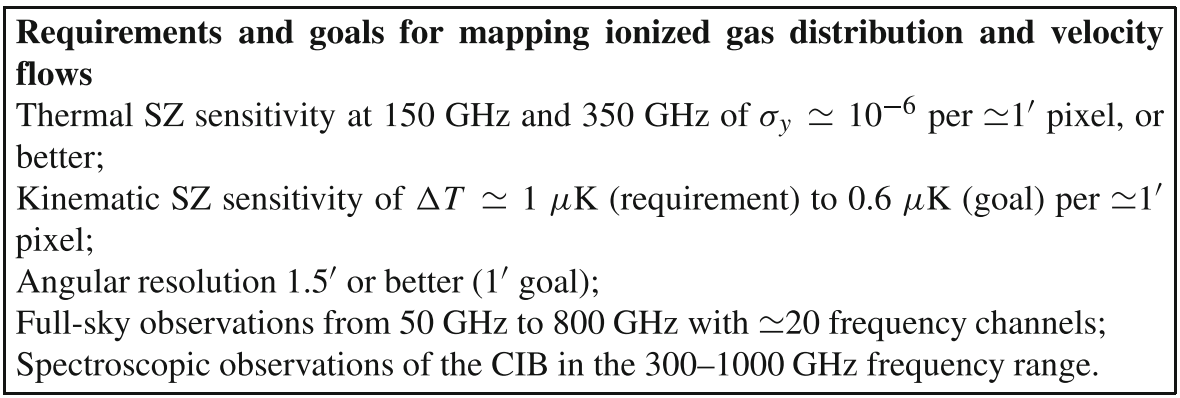

\subsection{A survey of matter through CMB lensing}

The lensing of the CMB by gravitational potentials along the line of sight is of considerable interest in two main regimes. First, very large-scale structures, for which the growth is still linear, generate deflections of the path of the photons with typical amplitude of $3^{\prime}$. These deflections are coherent over scales of a few degrees. Maps of the deflections can be used to reconstruct maps of the gravitational potential on the largest scales. The fidelity of such reconstruction is determined by the sensitivity to the lensing $B$ modes. ${ }^{4}$ If a survey's $B$-mode sensitivity is better than the level of the $B$-mode lensing signal ( $5 \mu \mathrm{K}$ arcmin amplitude), signal-dominated maps of the lensing potential can be reconstructed, down to $20^{\prime}$ for a CORE-like CMB polarization survey [24], and about $10^{\prime}$ or better with higher sensitivity, as in the case of PICO [25]. Secondly, on much smaller scales, dense, collapsed objects, such as massive clusters of galaxies, also deflect the CMB backlight. This can be used to estimate the mass of the lensing object, as illustrated on the right panel of Fig. 3.

\subsection{Survey requirements for CMB lensing}

The reconstruction of maps of integrated lensing potential and the calibration of the cluster masses require high-sensitivity observations of the CMB temperature and polarization, in particular on small scales. Lensing reconstruction can be performed

\footnotetext{
${ }^{4}$ The angular power spectra of the polarization of the CMB are encoded in terms of $E$ and $B$ modes. Inflation, density perturbations, and foregrounds produce $E$ modes. Inflation, lensing, and foregrounds each produce distinct pattern of $B$ modes.
} 

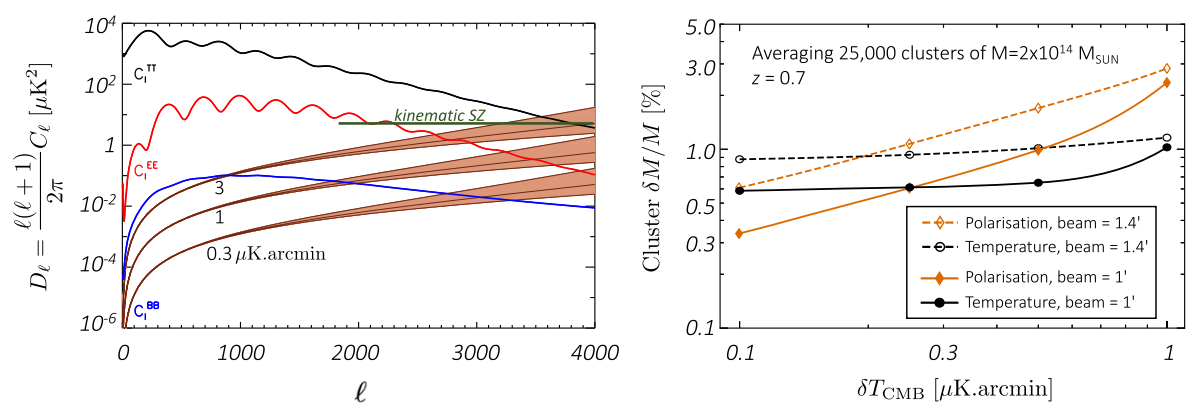

Fig. 3 Left: CMB $T T, E E$, and lensing $B B$ spectra. The light brown bands correspond to noise at the level of 3,1 , and $0.3 \mu \mathrm{K}$ arcmin, and angular resolution ranging from 1 to 3 arcmin. The dark green horizontal line shows the approximate level of the kSZ effect on small scales $(\ell>2000$, [26]). Right: Accuracy of cluster mass calibration achieved by averaging 25,000 clusters at redshift 0.7 , both from temperature and from polarization measurements, for resolutions of $1.4^{\prime}$ and $1^{\prime}$

with various combinations of CMB maps. The $T T$ estimator uses intensity only, while the $E B$ estimator, which is the most effective for very low noise, uses $E B$ cross-correlation [27].

Sensitivity and angular resolution It has been shown that in the case of a survey with $3 \mu \mathrm{K}$ arcmin $\mathrm{CMB}$ intensity sensitivity, the quality of the lensing reconstruction saturates for an angular resolution of $2-4^{\prime}$, while for a $4^{\prime}$ angular resolution survey, it saturates for a map sensitivity of the order of $0.1-0.3 \mu \mathrm{K}$ arcmin (see figure 4 of [27]).

Figure 3 illustrates how varying the sensitivity and angular resolution of a survey changes the performance of lensing measurements. As shown in the left panel, for a noise level of $3 \mu \mathrm{K}$ arcmin, lensing $B$ modes are dominant over the noise up to a limiting $\ell_{\text {lim }} \simeq 800$, independently of the angular resolution (here from $1^{\prime}$ to $3^{\prime}$ ). Hence, the number of harmonic modes that can contribute to lensing reconstruction is independent of the angular resolution for this instrumental sensitivity. When the sensitivity is increased to 1 or $0.3 \mu \mathrm{K}$ arcmin, $\ell_{\text {lim }}$ grows substantially, increasing the number of modes useful for lensing reconstruction with the EB estimator by a factor 4-10 (for lower noise, $\ell_{\lim }$ becomes dependent on the angular resolution).

At the same time, we note that the $\mathrm{kSZ}$ effect becomes the dominant source of confusion for lensing reconstruction with $T T$ when the noise level is below $3 \mu \mathrm{K}$ arcmin. This confirms the sensitivity requirement to measure the kSZ effect (Section 3.3), and shows that only polarization can improve lensing reconstruction when the performance of the instrument exceeds this level.

The right panel shows a confirmation from estimates of the accuracy of the calibration of cluster mass from stacking lensing constraints for a population of 25,000 clusters at $z=0.7$. The sensitivity saturates for a low noise level in $T T$, while the polarization constraints keep improving. We see from the right panel of Fig. 3 that if the sensitivity is at the level of about $1 \mu \mathrm{K}$ arcmin, an angular resolution of $1.4^{\prime}$ or better allows for calibrating cluster masses at about the $1 \%$ level with $T T$ only, 
which outperforms polarization constraints. With a sensitivity $0.6 \mu \mathrm{K}$ arcmin and a $1^{\prime}$ beam, we improve the constraint by a factor of 2 . To obtain comparable constraints with polarization (as a cross-check, and for an improvement by $\sqrt{2}$ of the overall sensitivity), we need a sensitivity better than $0.3 \mu \mathrm{K}$ arcmin, and a $1^{\prime}$ beam.

Frequency range and number of channels Requirements in terms of frequency range and frequency channels are similar for lensing as for measuring SZ effects (the kSZ effect has the same electromagnetic spectrum as the lensing signal).

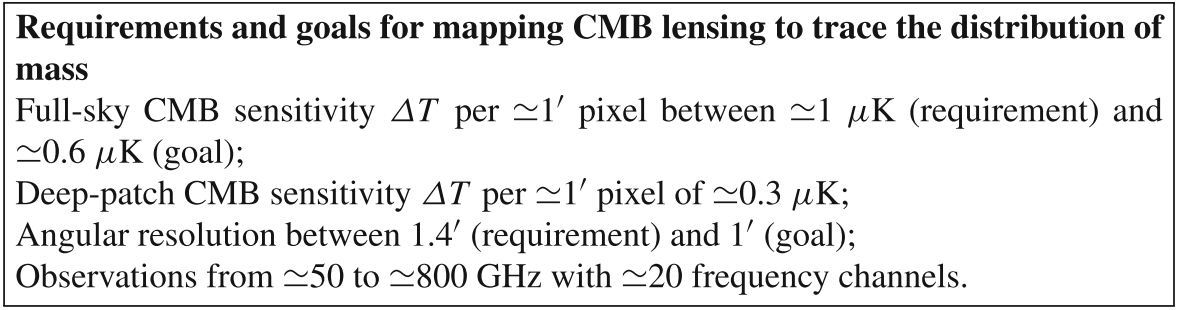

\section{High-redshift structures on the largest scales}

While the first stars reionize the Universe at redshift $z \simeq 8$, they also convert a fraction of the primordial hydrogen and helium into heavier atoms (metals), which then form molecules and dust particles that emit radiation at (sub)mm wavelength through thermal and line emission. Their detection out to high redshift, across large patches of sky, in hundreds of frequency bands, opens the path to a census of baryons in these various forms across cosmic time, and hence to the star formation history. Fluctuations of this emission trace the cosmic web at high redshift, and hence structures in a large fraction of the Hubble volume.

\subsection{Revealing galaxy protoclusters via dusty starbursts}

Understanding the full evolutionary history of galaxy clusters [28], the largest virialized structures in the Universe, is of fundamental importance for the observational validation of the formation history of the most massive dark-matter halos, a crucial test of models for structure formation, as well as for investigating the impact of environment on the formation and evolution of galaxies. Because of their deep potential wells, clusters may preserve fingerprints of the physical processes responsible for triggering and suppression of star formation and black-hole activity. Historically, clusters of galaxies have also been powerful probes of cosmological parameters.

Galaxy clusters in formation are called protoclusters [29]. Above $z \simeq 2$ protoclusters are found to be bright at far-IR/sub-mm wavelengths $[30,31]$ because a substantial fraction of their member galaxies, the dusty star-forming galaxy population (DSFGs, [32]), are undergoing intense, dust-enshrouded star-formation activity. 
They are rich in molecular gas and heavily obscured by dust. They are thus prime targets for sub-mm observational facilities.

Consider a 2-yr full-sky spectro-imaging survey with a 3.5-m telescope and a 64pixel filter-bank camera observing from $150 \mathrm{GHz}$ to $750 \mathrm{GHz}$ with $R=300$, an optical efficiency of $30 \%$, and instrumental noise close to the photon noise. Such an instrument has FWHM ranging from $2.5^{\prime}$ at $150 \mathrm{GHz}$ to $0.5^{\prime}$ at $750 \mathrm{GHz}$ and a $5 \sigma$ point source detection limit ranging from $5 \mathrm{mJy}$ at $750 \mathrm{GHz}$ to $28 \mathrm{mJy}$ at $150 \mathrm{GHz}$. The corresponding line detection limits are $5 \times 10^{-20} \mathrm{~W} \mathrm{~m}^{-2}$ and $1.4 \times 10^{-19} \mathrm{~W} \mathrm{~m}^{-2}$, respectively.

Such a spectroscopic survey can go deeper than the broadband Herschel surveys, with a similar telescope size, because they can take full advantage of the extreme sensitivity of state-of-the art instruments, and do not have the same confusion-noise limit as continuum surveys.

With the resolution of the example instrument, protocluster cores, having sizes of a few $100 \mathrm{kpc}$ [34-37], are unresolved clumps of DSFGs and so will show up as extremely bright sub-mm sources. We expect the detection of the strongest sub-mm lines for thousands of them all the way to the reionization epoch (see Fig. 4). At the peak of cosmic star-formation activity $(z=2-3)$ we expect the detection of hundreds of thousands protoclusters. At $z>2$ at least two lines will be detected, allowing a solid redshift determination without requiring follow-up observations.

No other foreseen survey can do anything similar. For example, Euclid will detect galaxy protoclusters up to $z \simeq 2.5$ and will miss those with strong obscuration by dust. The dynamics and chemistry of the discovered protoclusters can be further studied with ground-based large-aperture sub-mm telescopes and interferometers including the Atacama Large-Aperture sub-mm/mm Telescope (AtLAST) [38, 39], NOEMA and ALMA, underscoring the survey's complementary with these efforts.

As a by-product of our systematic search for protoclusters, we expect to identify tens of thousands of strongly lensed dusty starbursts [40] out to $z \simeq 7$ or even higher. Preliminary studies of this kind have been carried out for Planck-selected sources [41-44]. High-resolution follow up (aided by strong lensing) of such exceptionally bright galaxies will provide direct information on the complex physics that governs galaxy formation and early evolution [45].
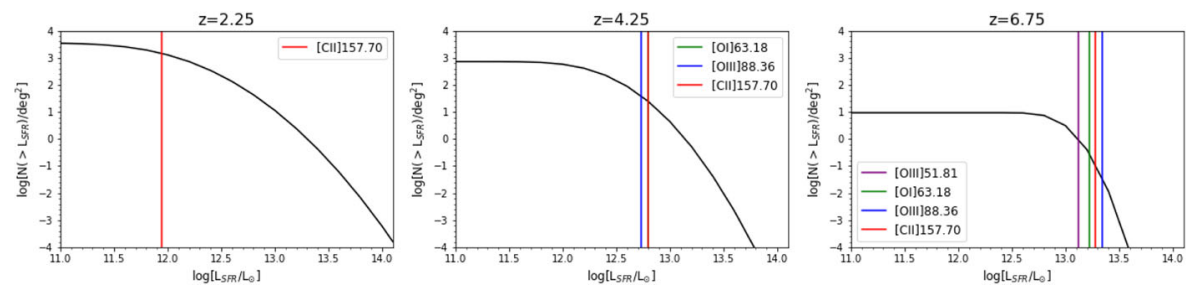

Fig. 4 Cumulative IR $(8-1000 \mu \mathrm{m})$ luminosity functions within $\delta z=0.5$ at three redshifts. The predictions are based on the model by Negrello et al. [33]. The line luminosities corresponding to $L_{\mathrm{IR}}$ were computed as described in the text. The vertical lines show the detection limits for the brightest lines, assuming the instrument-performances quantities described in the text. Such an instrument will detect protoclusters of dusty galaxies all the way out to the re-ionization redshift 


\subsection{Tomography of the cosmic infrared background}

The CIB is a major component of the extragalactic background light, with a spectrum that spans wavelengths from the millimeter regime down to the mid-infrared $[46,47]$. The CIB is sourced by emission from starlight-heated dust in galaxies with a young population of stars. Moderate resolution spectroscopy $(R \simeq 100)$ with wide frequency coverage can yield tomographic CIB maps that trace galaxy evolution across time.

Measurements of both the mean intensity and the spatial fluctuations of the CIB can yield important scientific output. Reaching high accuracy in absolute measurements is particularly challenging. Current constraints from the combination of absolute photometry from FIRAS with relative photometry from Planck are only at the $10 \%$ level [48]. A future space mission with an absolute spectrometer will allow more than an order-of-magnitude improvement in precision and enable the detection of extended intergalactic dust emission (or emission from more exotic sources such as dark-matter decay [49]), by separating out the signal coming from known galaxy populations.

Meanwhile, measuring the anisotropies in the CIB can be used to probe properties of the host halos of DSFGs [50]. However, this approach is only useful up to $z \simeq$ 3 due to large degeneracies between the effects caused by different sources of the anisotropy [51]. Therefore, at higher redshift it is more useful to use cross-correlation with other tracers of galaxy clustering and star-formation history, so that the redshift information is used to break the degeneracies. The CIB fluctuations signal can be fully exploited in cross-correlation with either spectroscopic galaxy surveys at low redshifts or line-intensity maps (e.g., of $\mathrm{CO}$ and $\mathrm{CII}$ emission) at medium and high redshifts. Cross-correlation with CMB lensing is also promising, particularly near the peak of the CMB lensing kernel, which roughly coincides with the peak of the starformation rate. CIB fluctuations can also provide an increase in the number of modes used in cosmological analyses at high redshift, improving the constraining power on primordial non-Gaussianity [52], for example.

\subsection{Mapping first stars and first metals}

An instrument targeting a wide range of frequencies with good spectral resolution can map the intensity fluctuations in multiple molecular and atomic lines across a correspondingly wide range of source redshifts. Line-intensity mapping (LIM) [53]—a measurement of spatial fluctuations in the integrated spectral-line emission originating from many individually unresolved galaxies and from the diffuse intergalactic medium-makes it possible to track the growth and evolution of cosmic structure at otherwise inaccessible redshifts.

Figure 5 shows targets for line-intensity mapping in the frequency range of interest. Notably, these include higher rotational transitions of the carbon-monoxide (CO) molecule, and the $158 \mu \mathrm{m}$ CII fine-structure line. The former provide excellent tracers of molecular gas evolution in galaxies, while the latter provides strong constraints on dust-obscured star formation, and is a key probe of galaxies during the epoch 


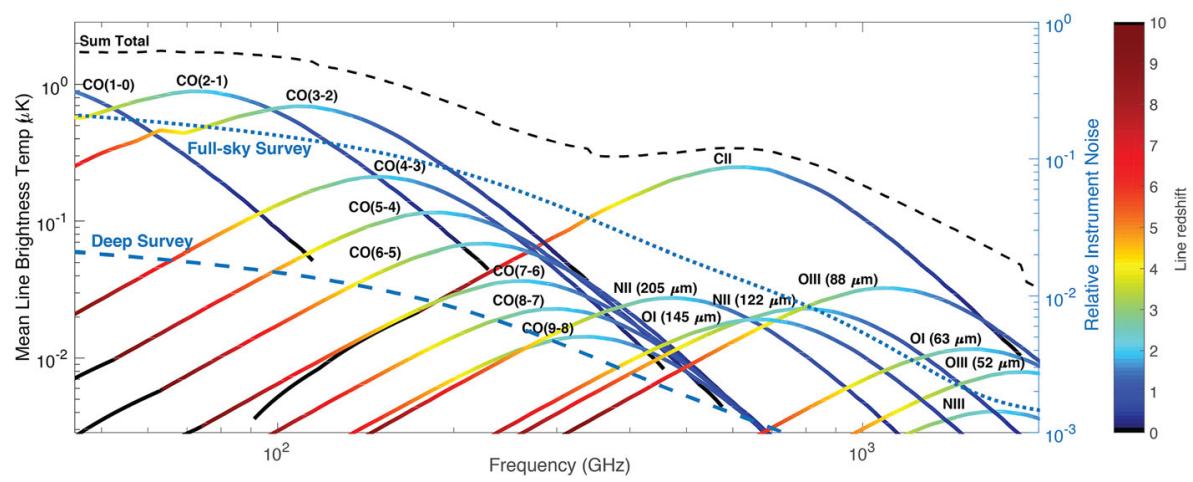

Fig. 5 Line emission from various extragalactic atoms and molecules. The sensitivity per $\mathrm{deg}^{2}$ expected from a 2-year full-sky survey (dotted blue line) and a deep intensity-mapping survey (dashed blue line) is also plotted. We assume 64 spectrometric pixels with $R=300$ at the focus of an 8-K, 3.5-m telescope, at the photon noise limit, with efficiency $30 \%$. The full-sky survey is expected to detect [CII] emission from dust heated by star formation with high $\mathrm{S} / \mathrm{N}$ out to $z \simeq 5$, and [OIII], [NII] at lower redshift. A deep survey can measure the CO ladder out to a redshift of 4 . The line strengths were modelled as a function of IR luminosity using observationally-based scaling relations [54, 55]. These measurements include local, and high-z galaxies; due to the lack of better constraints, the line ratios are assumed to be constant with redshift. The IR luminosities used to compute the line intensities, and bias was derived from the SFR in the Eagle (Evolution and Assembly of GaLaxies and their Environments) simulation [56], which constrains these relations as a function of redshift. The Star Formation Rate Density (SFRD) derived from the Eagle simulation shows a good fit to UV based observational constraints. Due to the lack of observational constraints, the modeling of these lines is uncertain by a factor of a few (low redshift) to an order of magnitude towards high redshift $(z>6)$. The modeling of these lines with other analytical calculations and galaxy simulations face similar problems due to a lack of constraints on a large number of free parameters (see e.g. [57]). These line models can only be meaningfully improved with a LIM space mission like the one proposed here

of reionization. Additional accessible lines include [NII] and [OIII] from stellar/HII regions, plus $[\mathrm{OI}]$ and $[\mathrm{CI}]$ from photo-dissociation regions (PDRs), among others.

Detection of multiple lines from the same structures is especially useful. First, this would allow a proper separation of the different line emission components contained in the intensity maps, which is essential to making robust interpretations of the measurements. Secondly, information from different lines can enhance the scientific output, e.g., probing the distribution of gas densities and temperatures in molecular clouds hosting star formation. At low $z$ the $\mathrm{CO}$ ladder can be used to constrain molecular gas with a much higher precision than with just one line, while at high $z$ the overall CO emission in high- $J$ transition lines can probe important properties such as gas turbulence, star-formation efficiency, metallicity, and the strength of the ionizing radiation field. Synergies between different lines can be used to characterize the physical processes that govern reionization, which span various scales and different regions within the first galaxies. In addition, the large-scale fluctuations in this emission should correlate with the overall morphology of the reionization field, and LIM of metal lines from the first galaxies will shed light on the timeline for metal and dust enrichment of the IGM. 


\subsection{Perspectives for cosmology with line-intensity mapping}

Until now, constraints on the parameters of $\Lambda \mathrm{CDM}$ have come from two main sources, the CMB and galaxy surveys, which originate from high and low redshifts. Over the wide intervening redshift range, between the last-scattering surface and the reach of galaxy surveys, we currently lack observables that provide low uncertainty measurements (Fig. 6). Measurements with LIM across this uncharted volume have the potential to increase cosmological parameter constraining power, and they hold unique qualitative advantages over the more established observables. For example, non-linear effects come in at smaller scales as we go to higher redshifts, allowing more robust comparison to theoretical calculations. Ultimately, the combination of experiments targeting different observables will be most effective in breaking different degeneracies between the cosmological parameters.

As well as improving $\Lambda \mathrm{CDM}$ constraints, LIM has the potential to shed light on the nature of dark matter, dark energy, and what drives early-Universe inflation. Intensity mapping of $\mathrm{CO}$ rotational lines at medium redshifts and $\mathrm{CII}$ emission towards reionization can fill the gap in measurements of the cosmic expansion history [58], which may be crucial for understanding the growing tension with measurements of the Hubble constant and whether it involves time-dependent dark energy. The large number of modes can yield constraints on primordial non-Gaussianity at the level of $f_{\mathrm{NL}} \lesssim 1$ [59], which is the target threshold for discerning between single and multi-field models of inflation. Mono-energetic dark matter decay can be tested using its correlation with the mass distribution inferred from the cross-correlation of spectral-intensity maps with galaxy or weak-lensing surveys [49]. And probing beyond $\Lambda \mathrm{CDM}$, powerful constraints can be placed on the number of effective relativistic degrees of freedom and the sum of neutrino masses [60].

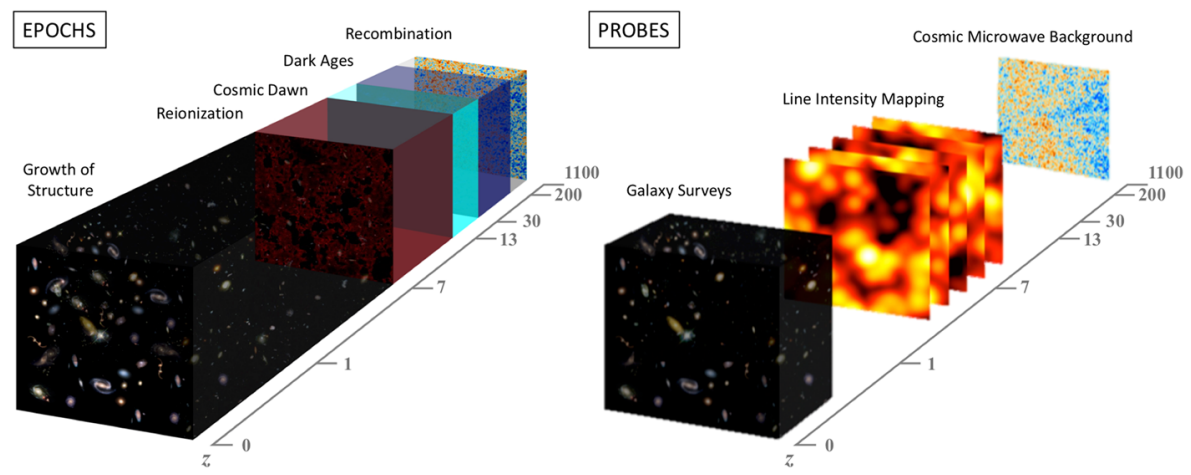

Fig. 6 Left: Various epochs in cosmic history, from CMB emission at the time of recombination, to present times. Right: $\mathrm{CMB}$ anisotropies map a shell of the Hubble volume located at $z \simeq 1100$. Line-intensity mapping (and CIB tomography) map the large-scale distribution of matter over a wide range of redshifts that cannot be easily accessed by any other means and are at higher $z$ than typical galaxy surveys 


\subsection{Survey requirements for high-redshift science}

The study of the high-redshift Universe as described above combines the detection of three types of emissions: i) diffuse dust emission from the background of unresolved galaxies constituting the CIB; ii) emission from unresolved galaxies with LIM of metal lines for mapping large scale structure (LSS) and its cosmic evolution; iii) emission from compact sources (continuum and lines) at high redshift.

Mapping diffuse emissions can be done with moderate angular resolution $\left(\sim 5^{\prime}\right)$. To map CII at $z>5$, up to the epoch of reionization, we need a sensitivity of $0.03 \mu \mathrm{K}$ at $200 \mathrm{GHz}$ to $400 \mathrm{GHz}$ (Fig. 5). The four first lines of the CO ladder can be mapped between $50 \mathrm{GHz}$ and $200 \mathrm{GHz}$ with sensitivity $0.2 \mu \mathrm{K}$. We require detecting at least two of them at each redshift bin together with CII so that we can separate the different lines.

As the level of these emissions is uncertain, we require that the space mission have the capability, in addition to the full sky survey, to map a deep patch of sky of a few hundred square degrees.

Detecting individual high redshift objects requires the best possible angular resolution. However, with LIM, even with a resolution of a few arcminutes a survey matching the above sensitivity requirements would detect many high redshift protoclusters and strongly lensed dusty galaxies [45].

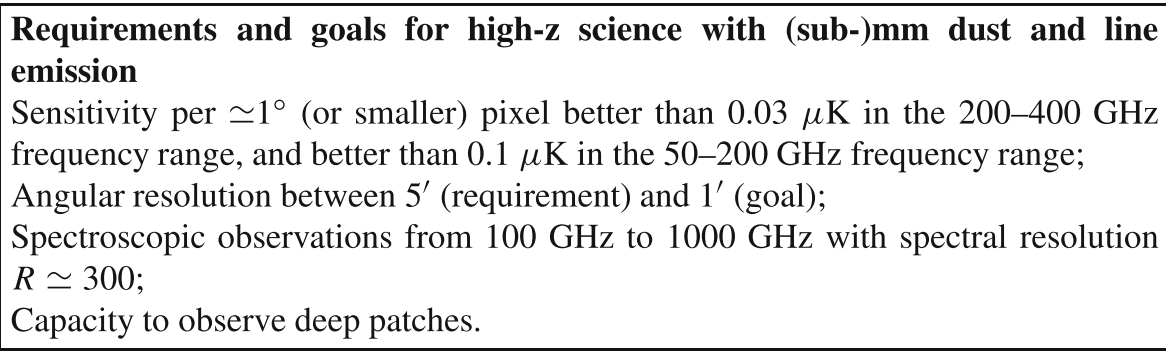

\section{Cosmology and fundamental physics}

With its measurements of the CMB Planck gave percent level constraints on seven $\Lambda C D M$ parameters. Although the science case of our proposed survey goes well beyond what can be achieved with observations of the primary CMB alone (see Appendix A.3), significantly more information about our Universe can also be extracted by a more sensitive CMB survey that has better angular resolution than Planck. Figure 7 shows the increase in the figure of merit (FOM) since COBE for the $\Lambda \mathrm{CDM}$ model (dark purple) and several extensions. Our proposed survey, like that of the PRISM mission concept [61] from which it is inspired, could almost reach the cosmic-variance limit. It would outperform what is expected from the combination of upcoming instruments, such as LiteBIRD for large angular scales [62], and Simons Observatory [63] or CMB-S4 [64] at higher angular resolution. 


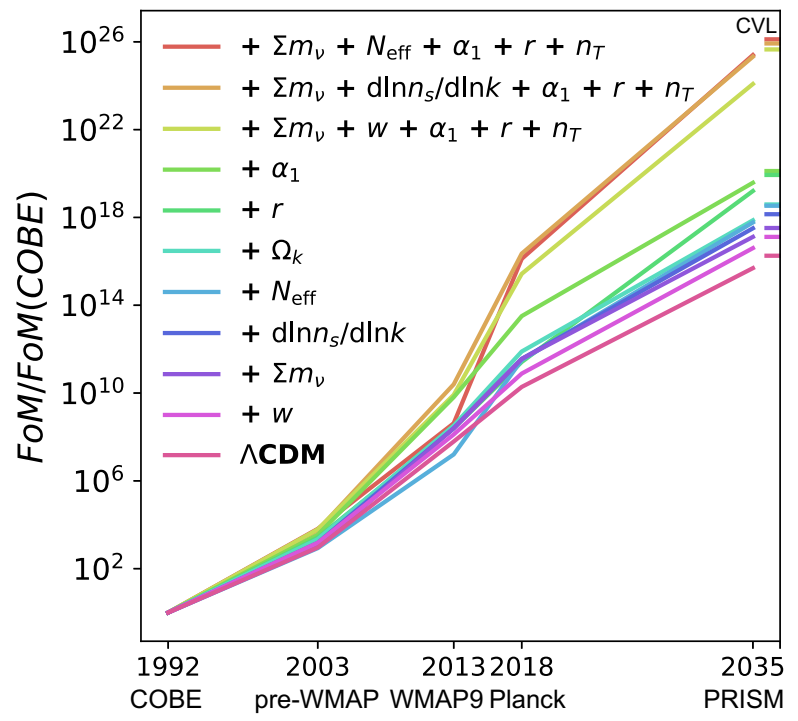

Fig. 7 FoM improvement since $C O B E$ for $\Lambda \mathrm{CDM}$ and several extended cosmological models. For PRISM in 2035, we consider an instrument with $P I C O$-like channels and sensitivity, and 2.5 times smaller beams. The constraints on $\Lambda \mathrm{CDM}$ and extensions reach the cosmic variance limit (CVL), shown as horizontal dashes on the right

\subsection{Gravitational waves and inflation}

CMB $B$-mode polarization is a unique window for detecting gravitational waves from inflation at an energy scale approximately a trillion times higher than those probed by the Large Hadron Collider. Similar to PICO [25], the proposed survey could also reach $\sigma(r) \sim \mathcal{O}\left(10^{-4}\right)$, approximately 300 times better than the current sensitivity $[65,66]$ and an order of magnitude below the uncertainty targeted by any single experiment in the next decade. Classes of inflationary models motivated by string theory or supergravity, predicting $r \lesssim 10^{-3}$, could be probed unambiguously. As an example, the Kahler geometry of $\alpha$-attractor models motivated by maximal supersymmetry [67, 68], could be probed entirely at high statistical significance. In the case of no detection, a vast class of large-field inflationary models will be ruled out.

The survey will measure the power spectrum of curvature perturbations with a combination of range of angular scales and precision that are unprecedented for a single experiment. The forecast uncertainty on the scalar spectral index $\sigma\left(n_{\mathrm{s}}\right) \lesssim 0.0015$ is more than a factor of three tighter than current measurements, and will reach the required precision to constrain the reheating stage after inflation for a given inflationary model. The forecast precision on the running of the spectral index is 0.0015 , at the same level as the theoretical predictions for single-field slow-roll inflationary models that provide a best-fit to Planck data, such as $R^{2}$ or Higgs inflation. The proposed survey will therefore have the capability to discriminate among different inflationary models also on the basis of the shape of the curvature of the power spectrum. 
Standard single-field slow-roll inflationary models predict primordial fluctuations with highly Gaussian statistics, compatible with the most recent Planck constraints on the local, equilateral, and orthogonal shapes of the bispectrum [69]. We can improve by a factor 2-3 on these bispectrum constraints, which are important to constrain models beyond the simplest ones, such as those with a non-trivial sound speed for the inflaton or with multiple fields. An enhanced sensitivity to the local shape of the bispectrum, down to $\sigma\left(f_{\mathrm{NL}}^{\text {local }}\right) \sim 1$, an important threshold for multi-field inflationary models, can be reached by a tomographic cross-correlation of the lensing potential with deep radio or photometric surveys in preparation, such as EMU, SKA or the Rubin Observatory (previously known as LSST).

\subsection{Neutrinos and extra relics}

Inferring the neutrino mass sum $M_{v}$ from cosmological data will remain a crucial target in the long term, since planned laboratory experiments are not sensitive to the minimal value $M_{v}=0.06 \mathrm{eV}$. Besides, it is important to exploit the synergy between cosmological surveys and laboratory searches, which are sensitive to different neutrino-related parameters and assumptions. On the cosmology side, precise measurements of $M_{v}$ require an exquisite mapping of both CMB anisotropies and large-scale structures (LSS). The two categories of observables are directly sensitive to the reduction in the growth rate of matter fluctuations induced by $M_{v}$, which $\mathrm{CMB}$ surveys probe through CMB lensing. CMB surveys will also play an essential role in accurately measuring other parameters like $\tau, n_{\mathrm{s}}, H_{0}$, and $\omega_{\mathrm{c}}$, that reduce degeneracies with $M_{v}$ in the analysis of LSS data. Our new survey alone will reach a sensitivity of $\sigma\left(M_{v}\right) \simeq 0.04 \mathrm{eV}$, and will be crucial in order to obtain $\sigma\left(M_{v}\right) \simeq \mathcal{O}\left(10^{-2}\right) \mathrm{eV}$ in combination with future galaxy, cosmic shear, and intensity-mapping surveys.

A plethora of extensions of the standard model of particle physics predict a relic density of extra light particles that would show up as an increase in the effective neutrino number $N_{\text {eff }}$ beyond its standard value of 3.046 [70]. Measuring $N_{\text {eff }}$ is thus crucial for particle physics. CMB anisotropies are the most sensitive probe of $N_{\text {eff }}$. The proposed survey will provide unprecedented sensitivity to $N_{\text {eff }}$, with $\sigma\left(N_{\text {eff }}\right)=0.022$ using temperature and polarization, and $\sigma\left(N_{\text {eff }}\right)=0.016$ in combination with lensing extraction. In absence of extra relics, the standard value 3.046 will be distinguished from 3.0 at the $2-3 \sigma$ level, which will offer an accurate test of the standard model of neutrino decoupling and electron-positron annihilation. The possibility that any new scalar boson decouples from the standard model at some temperature $T<10^{3} \mathrm{TeV}$ will be either established or excluded at the $1.5 \sigma$ level $(2 \sigma$ or $3 \sigma$ for a fermion or vector boson, respectively). A measurement compatible with 3.046 would prove with the same significance that no new particles have left thermal equilibrium between the decoupling of top quarks (at redshift $z \sim 10^{14}$ ) and today.

Our survey will also be very sensitive to additional effects caused by the small mass of possible light non-thermal sterile neutrinos (whose effect would be roughly equivalent to a combination of $M_{v}$ and $N_{\mathrm{eff}}$ ), or to non-standard interactions in the 
neutrino sector (that would modify the so-called neutrino drag effects, particularly visible on intermediate and small scales in the polarization spectrum).

\subsection{Requirements and goals for primary CMB science}

Several projects plan next-generation observations of CMB anisotropies, in particular primordial polarization B-modes. Among those, the LiteBIRD satellite [62] has been selected as JAXA's strategic L-class mission for a launch in 2027, and the CMB-S4 experiment [64] was approved for CD-0 by the DOE in the US and is awaiting NSF participation.

We foresee that the LiteBIRD and CMB-S4 surveys can be improved with a subsequent space mission in the following ways:

1. full-sky maps with sensitivity and angular resolution matching the CMB-S4 3\% sky patch;

2. isotropic maps (with no filtering along the scans);

3. capability of full-sky delensing;

4. capability to measure B-mode polarization with a sensitivity to $r$ of the level $\mathcal{O}\left(10^{-4}\right)$

5. capability to de-lens with different methods (from CMB and from CIB maps);

6. extended frequency coverage, in the sub-mm domain and between atmospheric windows.

Sensitivity and angular resolution The combination of LiteBIRD and CMB-S4 will reach an aggregated CMB sensitivity of $\simeq 2.5 \mu \mathrm{K}$ arcmin at $\simeq 1^{\prime}$ angular scale over $70 \%$ sky, and $\mathrm{a} \simeq 1 \mu \mathrm{K}$ arcmin sensitivity at $\simeq 1^{\prime}$ angular resolution in the $3 \%$ sky deep patch (although with uneven frequency coverage, and possibly anisotropic filtering of the maps from the ground-based instruments). With $\sigma_{r} \simeq 0.001$, both CMB-S4 and LiteBIRD would detect tensor modes at more than $5 \sigma$ if $r>0.005$. We propose a full-sky survey with an order of magnitude improvement in sensitivity $(\sim 10$ times lower noise spectrum, and $\sigma_{r} \sim 0.0001$ ), for redundant capability to detect $r \sim 0.001$ at more than $5 \sigma$ by internal delensing. An angular resolution of $\sim 5^{\prime}$ is adequate for most of the CMB science. However, there is added value to increased angular resolution $\sim 1^{\prime}$ to deliver an unprecedented measurement of the CMB damping tail in temperature and polarization, which would lead to a sensitivity to neutrino physics beyond the experiments of the next decade.

Frequency range and number of channels The survey should allow for near-fullsky foreground cleaning in both temperature and polarization. As argued above, and demonstrated with simulations in the CORE study [71], a sensitive polarized imager with $\sim 20$ frequency channels spanning a decade in frequency (from $60 \mathrm{GHz}$ to $600 \mathrm{GHz}$ ) or more is adequate for this task. However, simulations have shown that a wider frequency range improves the effectiveness of some component separation techniques. We hence follow the $P I C O$ design and target a frequency range from $20 \mathrm{GHz}$ to $800 \mathrm{GHz}$, as for the PICO study [25]. 
Control of systematics and redundancy High-sensitivity observations of CMB polarization require exquisite control of systematic effects. Space offers the best environment for this. Methods for systematics control have been developed and assessed in the context of the CORE study [72].

\section{Requirements and goals for primary CMB science \\ Full-sky CMB sensitivity of $\simeq 1 \mu \mathrm{K}$ arcmin; \\ Angular resolution $5^{\prime}$ requirement, and goal 1'; \\ $\simeq 20$ frequency channels in the $20-800 \mathrm{GHz}$ frequency range; \\ Demonstrated control of systematics effects.}

\subsection{Tests of homogeneity and isotropy}

Testing the apparent large-scale anomalies observed in CMB temperature maps calls for investigations of our Universe on the largest scales using other observables than $T$ [73] - our mission can do this with many different probes of the Hubble volume. An intriguing possibility is to measure the dipole of cosmic backgrounds other than the CMB to test that it is entirely due to our motion, as usually assumed [74]. Other possible tests of the statistics on scales comparable to the Hubble radius include the stationarity and large-scale modulation of lensing potential maps, CIB fluctuations, SZ cluster counts, and the measurement of local quadrupoles at various redshifts through the polarised SZ effect. A survey capable of measuring these signals would be unique in assessing the large-scale homogeneity and isotropy of our Universe. These go beyond tests based on CMB temperature and polarization maps, which probe our Universe in a single redshift shell, and are limited by cosmic variance.

\section{Information from the CMB's near-blackbody spectrum}

The precise shape of the CMB energy spectrum encodes new information that can be extracted using absolute CMB spectroscopy. At redshifts $z \gtrsim 2 \times 10^{6}$, thermalization processes are efficient and promptly restore a near perfect blackbody spectrum of the $\mathrm{CMB}$ if full thermal equilibrium was perturbed. However, at later epochs, starting a few months after the Big Bang, traces of energy-releasing or photon-injecting processes can be found by measurements of departures from a perfect blackbody spectrum. While classically CMB spectral distortions are described as a sum of $\mu$ - and $y$-type distortion signals [75-78], modern treatments of the problem have demonstrated that far more than just two numbers can be extracted (e.g, [79-82]). Measurements can constrain processes expected within $\Lambda \mathrm{CDM}$ including the damping of primordial perturbations and the recombination radiation, and open discovery space to the pre-recombination Universe which cannot be accessed directly any other way. $C O B E$-FIRAS still defines the long-standing benchmark for CMB spectral distortions, but several orders of magnitude of sensitivity improvements are in principle possible, as envisioned for PIXIE (e.g., [83]), the spectrometer of PRISM [84], and Super-PIXIE [85]. 


\subsection{Spectral distortions as a new test of inflation}

Fluctuations set up by inflation dissipate their energy through photon diffusion. This causes a CMB distortion signal that can be used to derive stringent constraints on the amplitude and shape of the primordial power spectrum at scales inaccessible to other probes [86-89]. A spectrometer like PIXIE could rule out excess power at wavenumbers $k \simeq 50 \mathrm{Mpc}^{-1}-10^{4} \mathrm{Mpc}^{-1}$ at the level of $P(k) \gtrsim 10^{-8}$ [89]. This would place novel constraints on a wide range of early-Universe models outside of standard slow-roll inflation, including features or inflection points in the potential, particle production, and waterfall transitions [90]. With 10 times better sensitivity (a fraction of a Jy/sr) a spectrometer could detect the expected $\Lambda \mathrm{CDM} \mu \simeq 2 \times 10^{-8}$ at the $3 \sigma$-level [90]; see Fig. 8. This would constrain inflation models, with a guaranteed target within standard slow-roll inflation. In combination with a future CMB imager, a spectrometer could improve the limits on the running of the spectral index by a factor of about $2[84,90]$. Primordial local-type non-Gaussianity could also be constrained using $\mu$-distortion anisotropies [91-93], providing an independent new probe of early-Universe physics [90].

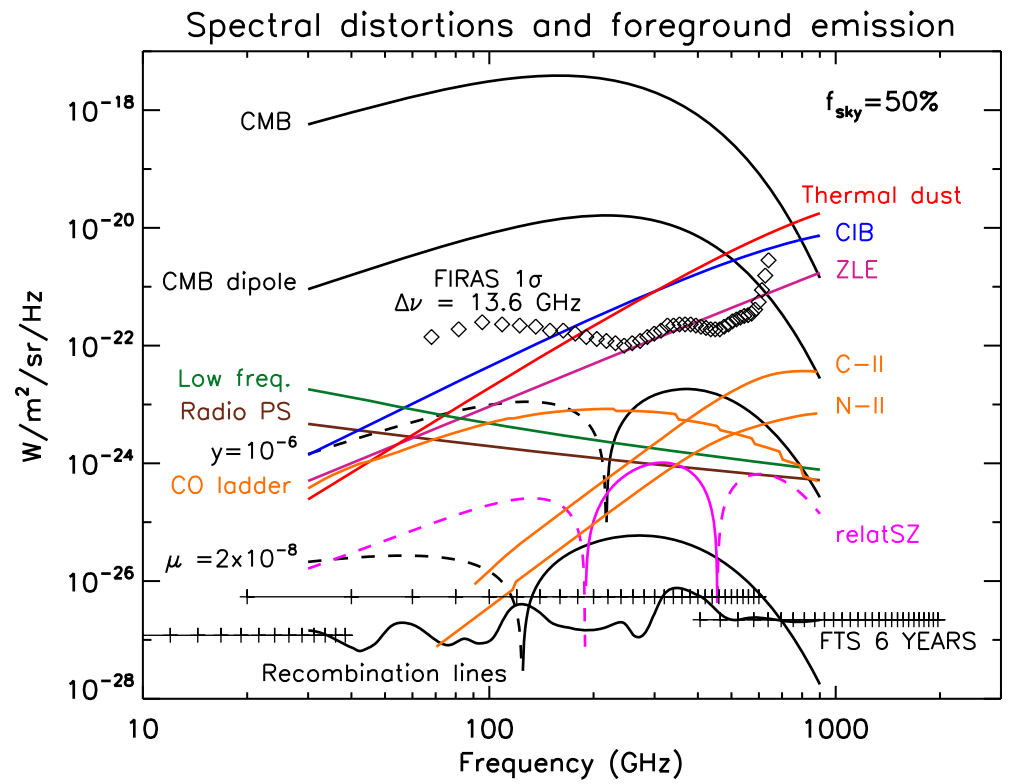

Fig. 8 The signals of interest that contribute to the total difference of microwave emission with a perfect blackbody span about 8 orders of magnitude in amplitude. The survey proposed here (indicated by black horizontal lines with vertical bars at the central frequencies) improves upon COBE-FIRAS (diamonds) by about four orders of magnitude, and would access the overall $y$-parameter signal (black dash and solid) discussed in section 2.1 and 5.2, as well as some of the signal from atomic and molecular lines discussed in section 3.3. It may make a few $\sigma$ detection of the $\mu$-distortion (black dash and solid) expected in $\Lambda \mathrm{CDM}$ and of the rSZ effect (magenta dash and solid), assuming that the total foreground emission can be measured and subtracted at the same level of accuracy 


\subsection{Reionization and structure formation}

The largest $\Lambda \mathrm{CDM}$ distortion is created by the low- $z$ structure formation and reionization process [94-97]. The first stars, accreting black holes, and shocks heat the baryons and electrons, which then up-scatter CMB photons to create an average $y$ type distortion. The overall expected distortion is $y \simeq$ few $\times 10^{-6}[95,98]$, one order of magnitude below the upper bound from $C O B E$-FIRAS. As shown in Fig. 8, this could be constrained to the sub-percent level with a future mission (see also [90]).

A large part of the low-redshift Compton-y signal is due to halos with masses $M \simeq 10^{13} \mathrm{M}_{\odot}$, which contain virialized gas with an electron temperature of $k T_{\mathrm{e}} \simeq 2-3 \mathrm{keV}$. This causes a relativistic temperature correction (rSZ) [99-101] that can directly tell us about feedback mechanisms [98]. Both the $y$ and the rSZ distortion depend directly on the shape and amplitude of the halo mass function, providing another cosmological measure of the growth of structure. With sufficient sensitivity, the survey could determine the average relativistic temperature with $\mathrm{S} / \mathrm{N}$ of tens-assuming foregrounds can be controlled-and constrain feedback physics that currently are still very poorly understood [90]. A direct measurement of the average rSZ temperature would also shed new light on the "missing baryon problem" [94] without the need to resolve the warm-hot-intergalactic medium. Measurements at $v \gtrsim 500 \mathrm{GHz}$ will probe the total cosmic-ray energy density of the Universe through the non-thermal relativistic SZ effect [90]. Furthermore, extremely precise spectrum measurements down to $\sim 10 \mathrm{GHz}$ will allow us to greatly improve our knowledge of the diffuse free-free emission associated with cosmological reionization, distinguishing between various models [102-104], and will shed light on the controversial question of a potential low-frequency background temperature excess raised by ARCADE 2 and EDGES results [105, 106]. These illustrate some of the unique opportunities in $\mathrm{CMB}$ spectroscopy.

\subsection{Probing dark matter and particle physics}

Dark matter is another example of how spectral distortions allow us to probe new physics. Non-baryonic matter constitutes $\simeq 25 \%$ of the energy density of the Universe, but its nature remains unknown. The long-favored WIMP-scenario is under increasing pressure [107-112], and emphasis is gradually shifting towards alternatives, prominent examples being axions, sterile neutrinos, sub-GeV Dark Matter (DM) or primordial black holes [113-119]. To solve this puzzle, a coordinated multitracer approach that combines different particle physics and cosmological probes is needed.

Measurements of the CMB anisotropies themselves have clearly helped to establish the presence of DM on cosmological scales and provided tight constraints on DM annihilation and decay [120-127] and interactions of DM with Standard Model (SM) particles [128-131]. However, for DM annihilation and decay CMB anisotropies quickly lose constraining power before recombination $\left(z \gtrsim 10^{3}\right)$, being impeded by cosmic variance. Similarly, measurements of light-element abundances [120, 132-134], which are only sensitive to non-thermal energy release above nucleardissociation thresholds in the pre-recombination era $[82,135]$, saturated their limits 
due to astrophysical uncertainties. This is where CMB spectral distortions offer a valuable complementary probe. For decaying particle scenarios, distortions are sensitive to particles with lifetimes $t \simeq 10^{6}-10^{12} \mathrm{~s}[79,82,136-140]$, providing a direct measurement of particle lifetimes via residual distortions [81, 82]. Similarly, annihilating particles can be constrained using distortions: $\mu$-distortions are sensitive to light particles ( $m \lesssim 100 \mathrm{keV}$ ) and complement $\gamma$-ray searches for heavier particles $[81,141]$. The rich spectral information added by various non-thermal processes [142-147] will allow us to glean even more information about the nature of dark matter.

This is new territory and more work is required; however, it is already clear that CMB spectral distortions can meaningfully probe scenarios involving axions [148$150]$, gravitino decays [137, 151], strings [152, 153], DM-SM-interactions [126, 154, 155], macroscopic DM [156], and primordial magnetic fields [157-160]. A CMB spectrometer that reaches the level of $\mu \simeq 10^{-8}$ after foreground marginalization can rule out a vast class of particle-physics models and also allow a first detection of the guaranteed $\mu$-distortion signal from the damping of primordial acoustic modes.

\subsection{The cosmological recombination radiation}

The cosmological recombination process causes another small but inevitable distortion of the CMB. Line emission from hydrogen and helium injects photons into the $\mathrm{CMB}$, which after redshifting from $z \simeq 10^{3}$ are visible today as complex frequency structure in the microwave bands [161-169]. The cosmological recombination radiation (CRR) has a simple dependence on cosmological parameters and the dynamics of recombination; since it includes not only hydrogen but also helium recombinations, it probes eras beyond the last-scattering surface observed by CMB anisotropies [170-172]. The signals are however weak and require noise levels of the order of 0.1 $\mathrm{Jy} / \mathrm{sr}$ or better.

\subsection{Requirements and goals for spectral distortion science}

Sensitivity and angular resolution $\mathrm{CMB}$ spectral distortion science is new territory, with a vast potential for discovery, but many unknowns on the path. For this reason, we set a relatively safe sensitivity requirement in terms of accessible science, i.e. an aggregated sensitivity at the level of $10^{-25} \mathrm{~W} / \mathrm{m} 2 / \mathrm{sr} / \mathrm{Hz}(10 \mathrm{Jy} / \mathrm{sr})$, sufficient to detect the mean $y$ level and high-redshift spectral lines plotted in Fig. 8. As a goal, we target 100 times better sensitivity, to look also for the faintest spectral distortion signals down to the $\mathrm{CRR}$. An angular resolution of $\simeq 1^{\circ}$ is sufficient for selecting data from relatively clean regions of the sky in terms of galactic and zodiacal foreground contamination.

Frequency range and spectral resolution The survey must cover the region where the specific spectral signatures of the distortions make them distinguishable from other emissions. We require $30-600 \mathrm{GHz}$ coverage, with a goal of $10-2000 \mathrm{GHz}$. A spectral resolution corresponding to $R \simeq 10$ is adequate to distinguish the various components. 


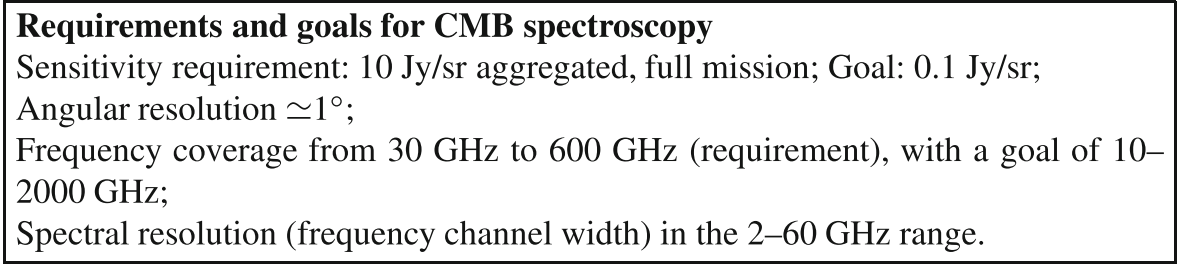

\section{Possible mission profiles}

Summarizing the requirements detailed above, this science program requires detecting the following signals with high signal-to-noise ratio and high precision, over the entire sky.

1. Thermal SZ emission from most galaxy clusters in the Hubble volume, to map hot ionized gas in the cosmic web: angular resolution 1.5 to $1^{\prime}$; SZ sensitivity $\Delta_{y} \simeq 10^{-6}$ at $1 \sigma$ per arcmin pixel around $150 \mathrm{GHz}$ and $350 \mathrm{GHz} ; \simeq 20$ frequency channels in the $50-800 \mathrm{GHz}$ frequency range.

2. $\mathrm{CMB}$ anisotropies generated by lensing effects and the kSZ effect; angular resolution 1.5 to $1^{\prime}$; CMB sensitivity $\Delta_{T} \simeq 1$ to $0.6 \mu \mathrm{K}$ arcmin; $\sim 20$ frequency channels covering the $50-800 \mathrm{GHz}$ frequency range.

3. $\mathrm{CMB}$ anisotropies from $z \simeq 1100$; angular resolution $5^{\prime}$ to $1^{\prime}$; sensitivity $\Delta_{T} \simeq$ $1 \mu \mathrm{K}$ arcmin; $\sim 20$ frequency band in the $20-800 \mathrm{GHz}$ frequency range.

4. Absolute emission and fluctuations from dust continuum and [CII]/CO lines across a wide range of redshifts (up to $z \simeq 10$ ) with spectral resolution $R=300$ extending from $\sim 100 \mathrm{GHz}$ to $\sim 1000 \mathrm{GHz}$; angular resolution $1-5^{\prime}$; capability to map deep patches.

5. Absolute spectrum of the microwave sky emission from $10 \mathrm{GHz}$ to $2000 \mathrm{GHz}$; angular resolution $\sim 1^{\circ}$; sensitivity integrated over the full observing time in the $0.1-10 \mathrm{Jy} \mathrm{sr}^{-1}$ range.

Overall, the goal would be to achieve, with a combination of instruments, a spectro-polarimetric survey of the entire sky from $10 \mathrm{GHz}$ to $2000 \mathrm{GHz}$, with angular resolution $1^{\prime}$ to $1.5^{\prime}$, and sensitivity matching the requirements of the above science goals.

\subsection{Mission overview}

The space mission should perform those necessary observations that cannot be done better from the ground. The key design elements are below.

- Angular resolution between $1.5^{\prime}$ and $1^{\prime}$ at $\geq 300 \mathrm{GHz}$. This requires a telescope with aperture between $2.8 \mathrm{~m}$ (requirement) and $4.2 \mathrm{~m}$ (goal). We rely on groundbased telescopes for the smallest scales at lower frequencies. A larger telescope in space would be challenging and not cost-efficient. 
- Two focal-plane instruments: a broad-band polarized imager from $20 \mathrm{GHz}$ to $800 \mathrm{GHz}$ for $\mathrm{CMB}$ anisotropies, $\mathrm{tSZ}, \mathrm{kSZ}$, lensing, and a spectrometer with $R \simeq$ 300 for CIB tomography and line intensity mapping from $100 \mathrm{GHz}$ to $1000 \mathrm{GHz}$.

- A set of FTSs covering 10-2000 GHz for measurement of the absolute spectrum.

This requires an L-class mission, or a combination of $\mathrm{L}$ and $\mathrm{M}$ missions. Such a mission, or combination of missions, could be envisaged in the early 2030s in the context of an international collaboration, e.g. between NASA and ESA. Downscoping to M-class would require reducing the telescope size by a factor $\sim 3$, and possibly relaxing the temperature requirement for the primary mirror, for a space mission similar to CORE or PICO. This would significantly degrade most of the high resolution science (cluster and protocluster surveys, CMB lensing science, velocity flows from kSZ), but remains appealing for primary CMB science (primordial gravitational waves, cosmological parameters constraints), as shown in the context of the CORE and PICO studies. Additional elements about the comparison of our proposed mission with existing and planned experiments are given in Appendix A.1, and a short discussion of costs, based on comparisons with other space missions and on previous studies, in Appendix A.2.

\subsection{Instruments}

Polarimetric imager The polarimetric imager must observe the polarized emission in several broad bands $(\Delta v / v \simeq 0.25)$ covering the frequency range at which $\mathrm{CMB}$ anisotropies and SZ effects dominate. Its science goals can be achieved with an instrument that is a straightforward extension of today's technologies, specifically based on the design for PICO, an instrument proposed for consideration by the US2020 decadal panel [25]; see Fig. 9. Table 1 gives the expected performance for a full-sky survey and a deep patch, observed for 2 years and 6 months, respectively, using this instrument.

The current telescope design for PICO, which has a 1.4-m diameter aperture, allows for a factor 2 increase to a diameter of $2.8 \mathrm{~m}$ and the instrument would fit with no other changes (except for the need of deployable shields) within the Ariane 6 shroud. Changes to the optical design should allow increase to diameters between $3.5 \mathrm{~m}$ and $4 \mathrm{~m}$. The Herschel mission had a 3.5-m telescope.

The focal plane is continuously maintained at $0.1 \mathrm{~K}$. Several technologies including continuous adiabatic-demagnetization refrigerators [173, 174] and continuouscycle dilution refrigerators [175] are either already near-mature, or should be mature by the 2030s. The focal plane module contains four elements: (1) low-pass filters rejecting electromagnetic (EM) radiation above the highest band $(\sim 850 \mathrm{GHz}) ;(2)$ monolithic arrays of thousands of pixel elements that couple the EM radiation from space to transmission lines, which then channel the power to transition-edge-sensor (TES) bolometers converting the deposited power to current signals; and (3) frontend SQUID amplifiers. Current technologies allow coupling a broad-band of EM radiation into a focal plane pixel using broad-band antennas or horns [176, 177], and then channeling specific frequency bands into their bolometers using on-wafer filters. We will use these technologies with up to three bands per pixel for frequencies 

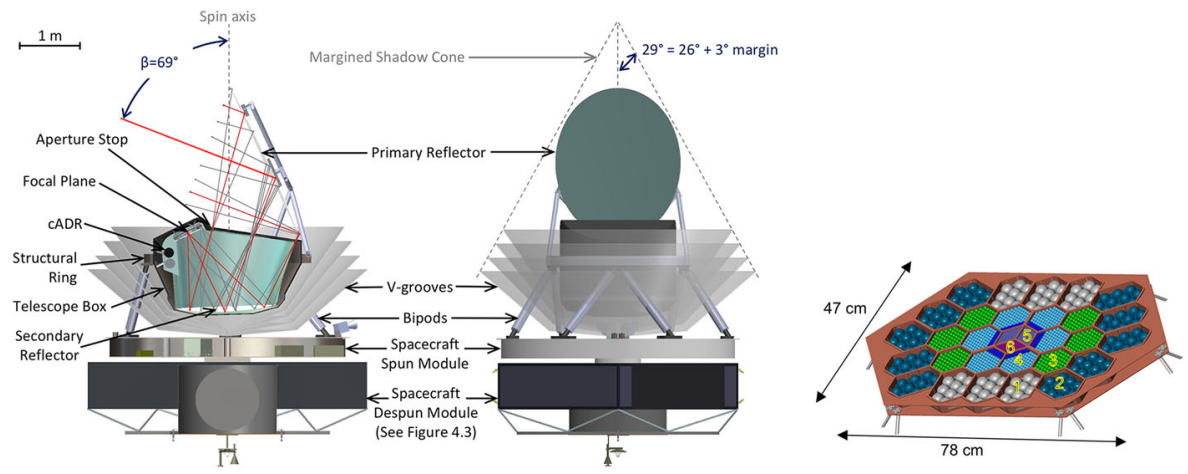

Fig. 9 PICO overall configuration in side view and cross section (left), front view with V-Groove assembly shown semi-transparent (middle), and the focal plane (right) (reproduced with permission from Hanany et al. [25]). A 2.8-m entrance aperture is achievable by scaling the PICO optical design by a factor of two and using deployable shields. The PICO focal plane (right), which contains 12,996 TES bolometers, achieves the noise levels baselined for the survey proposed here

up to $\sim 450 \mathrm{GHz}$, which is close to the $\mathrm{Nb}$ bandgap. At higher frequencies, for which we cannot use superconducting $\mathrm{Nb}$ to channel the EM into transmission lines, we will use direct absorption onto polarization-sensitive bolometers with one frequency band per pixel.

Both antenna-based and horns-based EM coupling of the radiation are polarization preserving; they do not alter the incident polarization, but can select for detection one of the polarization states. End-to-end polarimety is achieved by splitting the incident radiation into two orthogonal polarization states, and arranging the focal plane to have sensitivity to at least three orientations [25].

The readout is based on multiplexing $N$ detectors onto two readout lines (where $N$ is called the multiplexing factor). Both time-domain-based and frequency-domainbased multiplexing are in use by operating experiments. For PICO we assumed a conservative factor of 128. Systems with multiplexing factors between 2000 and 4000 are in development.

Absolute spectrophotometry Order-of-magnitude improvements to current upper limits for $\mathrm{CMB}$ spectral distortions require continuous spectra at modest spectral resolution, covering 6 or more octaves in frequency with part-per-million channel-tochannel calibration stability. Fourier transform spectroscopy is ideally suited to this task. The FTS measures the difference spectrum between the sky and a blackbody calibrator. Unlike an imager, whose frequency channels are set by bandpass filters, the central frequency, channel bandwidth, and channel-to-channel covariance of an FTS's synthesized frequency channels depend only on the sampling (apodization) of the interferograms and can be determined a priori. The photon noise to first order is the same for all channels; it depends on the integrated optical load over the total passband and scales linearly with the synthesized channel width.

A broad-band FTS based on the PIXIE design [83] would achieve the science goals outlined above. PIXIE uses a single FTS with $15-\mathrm{GHz}$ channels extending 
Table 1 Performance forecast for the polarized imager, from estimated performance of the instrument in the $P I C O$ study [25], and 3.5-m aperture optics

\begin{tabular}{|c|c|c|c|c|c|c|c|c|c|c|}
\hline $\begin{array}{l}v \\
(\mathrm{GHz})\end{array}$ & $\begin{array}{l}\text { Beam } \\
(\operatorname{arcmin})\end{array}$ & $\begin{array}{l}\mathrm{CMB} \sigma_{I} \\
(\mu \mathrm{K})\end{array}$ & $\begin{array}{l}\mathrm{tSZ} \sigma_{y} \\
\left(\times 10^{6}\right)\end{array}$ & $\begin{array}{l}\sigma_{I} \\
\left(\mathrm{kJy} \mathrm{sr}^{-1}\right)\end{array}$ & $\begin{array}{l}\text { PS } 5 \sigma \\
(\mathrm{mJy})\end{array}$ & $\begin{array}{l}\mathrm{CMB} \sigma_{I} \\
(\mu \mathrm{K})\end{array}$ & $\begin{array}{l}\mathrm{tSZ} \sigma_{y} \\
\left(\times 10^{6}\right)\end{array}$ & $\begin{array}{l}\sigma_{I} \\
\left(\mathrm{kJy} \mathrm{sr}^{-1}\right)\end{array}$ & $\begin{array}{l}\text { Flux } \times 10^{-20} \\
\left(\mathrm{~W} \mathrm{~m}^{-2}\right)\end{array}$ & $\begin{array}{l}\text { PS 5o } \\
(\mathrm{mJy})\end{array}$ \\
\hline 21 & 15.36 & 18.41 & -3.41 & 0.24 & 27.89 & 8.23 & -1.52 & 0.11 & 13.1 & 12.47 \\
\hline 25 & 12.8 & 12.88 & -2.4 & 0.24 & 19.12 & 5.76 & -1.07 & 0.10 & 10.69 & 8.55 \\
\hline 30 & 11.32 & 8.74 & -1.64 & 0.23 & 14.51 & 3.91 & -0.73 & 0.10 & 9.73 & 6.49 \\
\hline 36 & 9.44 & 6.13 & -1.16 & 0.23 & 10.09 & 2.74 & -0.52 & 0.10 & 8.12 & 4.51 \\
\hline 43 & 8.88 & 6.13 & -1.18 & 0.33 & 12.56 & 2.74 & -0.52 & 0.14 & 12.08 & 5.62 \\
\hline 52 & 7.35 & 4.29 & -0.84 & 0.33 & 8.64 & 1.92 & -0.37 & 0.14 & 10.05 & 3.86 \\
\hline 62 & 5.12 & 4.14 & -0.84 & 0.44 & 5.57 & 1.85 & -0.37 & 0.19 & 7.72 & 2.49 \\
\hline 75 & 4.27 & 3.22 & -0.68 & 0.48 & 4.23 & 1.44 & -0.30 & 0.21 & 7.10 & 1.89 \\
\hline 90 & 3.8 & 2.14 & -0.49 & 0.43 & 3.01 & 0.96 & -0.22 & 0.19 & 6.06 & 1.34 \\
\hline 108 & 3.16 & 1.68 & -0.43 & 0.45 & 2.16 & 0.75 & -0.19 & 0.20 & 5.21 & 0.96 \\
\hline 129 & 2.96 & 1.68 & -0.51 & 0.57 & 2.39 & 0.75 & -0.22 & 0.25 & 6.91 & 1.07 \\
\hline 155 & 2.48 & 1.38 & -0.56 & 0.56 & 1.67 & 0.61 & -0.25 & 0.25 & 5.79 & 0.74 \\
\hline 186 & 1.72 & 3.06 & -2.40 & 1.42 & 2.02 & 1.37 & -1.07 & 0.63 & 8.42 & 0.90 \\
\hline 223 & 1.44 & 3.52 & 15.29 & 1.70 & 1.69 & 1.57 & 6.84 & 0.76 & 8.45 & 0.75 \\
\hline 268 & 1.28 & 2.3 & 1.05 & 1.02 & 0.8 & 1.02 & 0.46 & 0.45 & 4.83 & 0.36 \\
\hline 321 & 1.04 & 3.22 & 0.69 & 1.15 & 0.59 & 1.44 & 0.31 & 0.51 & 4.28 & 0.26 \\
\hline 385 & 1.00 & 3.52 & 0.46 & 0.84 & 0.4 & 1.57 & 0.20 & 0.37 & 3.47 & 0.18 \\
\hline 462 & 0.84 & 6.90 & 0.61 & 0.87 & 0.29 & 3.08 & 0.27 & 0.39 & 3.07 & 0.13 \\
\hline 555 & 0.60 & 35.29 & 2.24 & 1.81 & 0.31 & 15.78 & 1.00 & 0.81 & 3.89 & 0.14 \\
\hline 666 & 0.52 & 136.5 & 6.48 & 2.06 & 0.26 & 61.07 & 2.89 & 0.92 & 3.98 & 0.11 \\
\hline 799 & 0.44 & 807.1 & 29.41 & 2.43 & 0.22 & 360.96 & 13.15 & 1.08 & 4.03 & 0.10 \\
\hline Total & & 0.66 & 0.17 & & & 0.29 & 0.077 & & & \\
\hline
\end{tabular}

The first set of sensitivity columns are for a 2-year full-sky survey, the next five for a deeper patch of $5 \%$ sky observed for a total of 6 months. Sensitivities for $y$ in the negative part of the tSZ spectrum are conventionally noted with a negative sign. $\mathrm{CMB}, y$, and brightness sensitivities are for $1^{\prime}$ pixels, while flux sensitivities (in $\mathrm{W} \mathrm{m}^{-2}$ ) and point source sensitivities (in $\mathrm{mJy}$ ) are integrated in the beam. Polarization sensitivities are obtained by multiplying these numbers by $\sqrt{2}$. The last line gives the aggregated focal plane array sensitivity to signals with the color of CMB or tSZ (actual sensitivity will be reduced after separation of the astrophysical components)

from $15 \mathrm{GHz}$ to $6 \mathrm{THz}$. If foregrounds were negligible, PIXIE could detect the $\mu$ distortion from Silk damping of primordial density perturbations at 2-3 $\sigma$ and detect recombination lines at comparable levels. Astrophysical foregrounds degrade the ideal performance and require additional sensitivity at both low and high frequencies [178].

Such sensitivity could be obtained using several nearly-identical FTS modules, each with different optical passbands and synthesized frequency channels, optimized 
for either the $\mathrm{CMB}$ distortion signals or for measuring the competing foreground emission. Table 2 shows the performance for a design with three modules covering, respectively, low, middle, and high frequencies (inspired from [85]).

Each module is based entirely on existing technologies.

Filter-bank spectrometer The recently demonstrated on-chip filter-bank spectrometer is an ideal candidate to provide spectral filtering and radiation detection over large bandwidths with minimal weight and complexity [179]. As shown in Fig. 10, it consists of a chip fabricated from an NbTiN superconducting film that creates an electrical circuit which combines radiation detection by means of an antenna, spectral filtering by a filter-bank spectrometer and detection by using background limited MKID detectors and their readout [180]. The technology proposed in Ref. [179] is intrinsically limited to a $90 \mathrm{GHz}$ to $1.1 \mathrm{THz}$ band due to the properties of the materials used, but can be easily upgraded to ultra-large bandwidths using leaky-wave antennas [181]. Developments using low-Tc superconductors (such as Ti or TiN) are needed to go down to $50 \mathrm{GHz}$; a dielectric-based filter-bank, taking advantage of the low loss tangents of crystalline $\mathrm{Si}$, is needed to extend the frequency range to $2 \mathrm{THz}$.

Other instrumental options Other options can be considered for achieving the proposed spectroscopic survey. An FTS in the focal plane could be implemented by means of a steerable mirror reorienting the beam towards the different instruments. This solution is attractive for spectroscopy at high angular resolution, with the price of complexity for the focal plane assembly. This solution is not well suited for very accurate measurement of CMB spectral distortions, which do not require high angular resolution, but must compare the sky to a movable (and tunable) calibrator, and require excellent stray-light control. In the focal plane of a large telescope, a planar FTS would be an interesting option, and easier to integrate close to other instruments. This kind of device, not ready yet, makes use of superconducting-microstrip or coplanar delay lines, currently investigated by several groups. Being coupled to planar antennas, they would be polarization sensitive, and inherently single mode. For line spectroscopy a Fabry-Perot interferometer and a grating spectrometer would be interesting options. A cold grating coupled to an actively cooled telescope meets the condition of the lowest photonic background for sub-mm line search, but it size scales linearly with the spectral resolution.

Table 2 Multi-module absolute spectrometer; The mission sensitivity in the last column assumes $70 \%$ useful data and a 6-year mission

\begin{tabular}{llllll}
\hline Module & $v_{\min }(\mathrm{GHz})$ & $v_{\max }(\mathrm{GHz})$ & $\Delta v(\mathrm{GHz})$ & Sensitivity $(\mathrm{Jy} \cdot \sqrt{\mathrm{s}})$ & Mission sens. $\left(\mathrm{Jy} \mathrm{sr}^{-1}\right)$ \\
\hline LFM & 9.6 & 38.4 & 2.4 & 1435 & 0.12 \\
MFM & 20 & 600 & 20 & 6200 & 0.54 \\
HFM & 406 & 2000 & 58 & 2520 & 0.22 \\
\hline
\end{tabular}



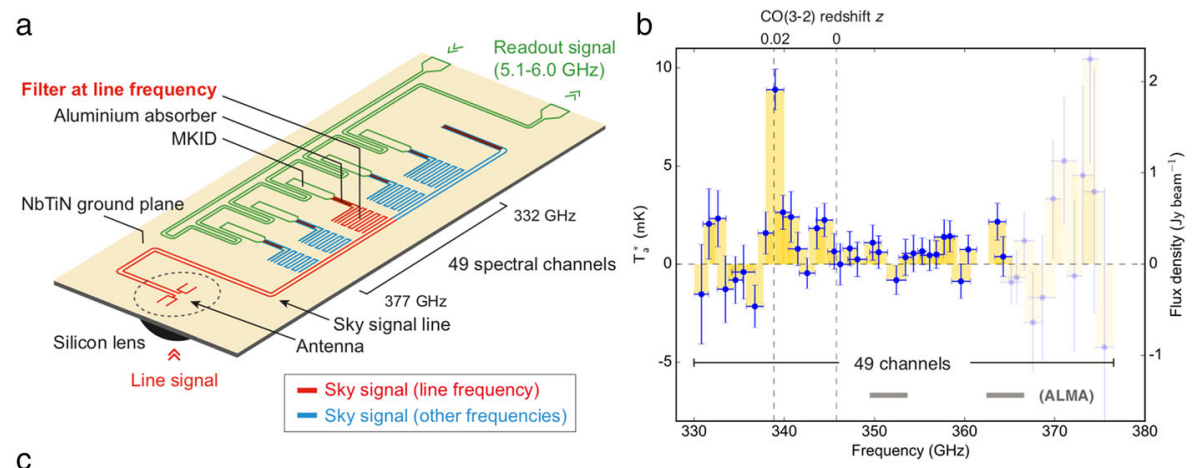

C

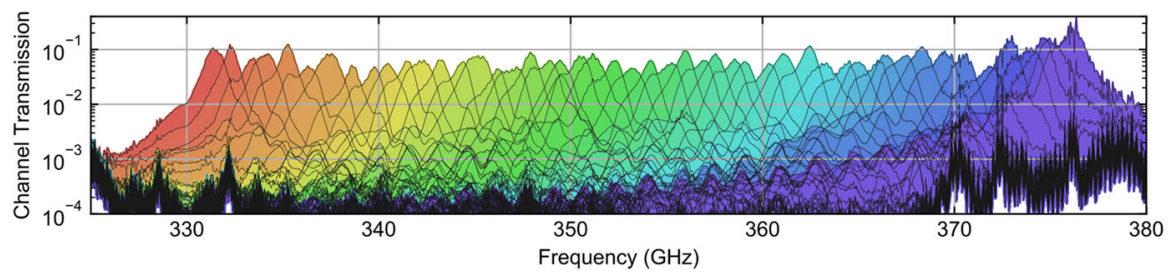

Fig. 10 a Sketch of the spectrometer chip. b Measured spectrum from VV114 with 2017 prototype. c Lab calibration of the spectral response of the individual filters. Reproduced with permission [179]

\section{Scientific and technological roadmap}

\subsection{Scientific heritage and complementarity of probes}

The roadmap to the proposed mission benefits from intermediate projects that address a fraction of the science case.

- LiteBIRD plans to observe CMB polarization on large angular scales to search for inflation-produced gravitational waves. Our proposed survey is designed for other scientific objectives, but still improves upon the LiteBIRD target search limits by a factor of 10 .

- The Simons observatory, and later CMB-S4, will observe the CMB and galaxy clusters with $1-1.5^{\prime}$ angular resolution in specific atmospheric windows. Our proposed survey, conducted within a single space mission, will be as sensitive as ten CMB-S4 experiments. The survey will complement the frequency coverage in the gaps between atmospheric windows, and make observations above $300 \mathrm{GHz}$ with angular resolution matching that of the ground experiments. This complementarity will be key to separate the mixture of SZ effects and CIB emission into their individual contributions.

- Data from other cosmological probes, most notably the Rubin Observatory (previously, LSST), will tighten constraints on the cosmological model; Consistency tests using data from different probes are essential for establishing confidence in a cosmological model. Complementary probes also help lift parameter degeneracies. 
Our proposed survey builds on the technological developments currently ongoing for the next generation experiments in CMB observations and line intensity mapping.

\subsection{Technology challenges and readiness}

The proposed survey does not require the development of technologies or techniques that have not been already demonstrated at some level, either in space, or in ground-based experiments. The main challenges are to scale up existing capability (in particular the number of detectors and frequency range of operation), to demonstrate flight readiness for all components, and to provide the scientific payload with all the necessary resources, in particular in terms of cooling of the telescope and of the focal plane, on-board power, and telecommunication for data download from an orbit around L2.

A 4-m class telescope at $8 \mathrm{~K}$ The baseline telescope has a 3.5-m aperture, and is actively cooled to $\simeq 8 \mathrm{~K}$. It is the same size used by Herschel, albeit much colder. One option proposed for the Origins Space Telescope [182], a mission proposed in the US for implementation in the 2020s, has a 5.9-m telescope cooled to $4 \mathrm{~K}$. For optimal science, a $20 \%$ increase of the aperture size with respect to the baseline ( $4.2 \mathrm{~m}$ instead of $3.5 \mathrm{~m}$ ) would be desirable if the capacity of the fairing of a next-generation European launcher allows it. Launchers with an 8-m fairing, as foreseen for the launch of the Origins Space Telescope flagship mission, are available in the US. Although not desirable, the aperture size could be reduced by $20 \%(2.8 \mathrm{~m})$, for an angular resolution of $1.4^{\prime}$ at $300 \mathrm{GHz}$. For comparison, the SPICA space mission, currently in a study phase, is planned to have a $2.5-\mathrm{m}$ primary actively cooled to $<10 \mathrm{~K}$.

Optical components Optical components for $\mathrm{mm}$ and sub-mm astronomy are widely available today. Devices and modeling tools are already reliable for various optical configurations (reflective or refractive). Polarization filtering and modulation, spectral filtering, phase control, and stray-light suppression are well understood and can be implemented in a variety of ways, also at cryogenic temperatures. For instruments to be launched post-2035, we can expect reduction in mass and increased compactness. Solutions based on artificial materials (metamaterials, photonic crystals) are progressing rapidly and will soon offer new tools for astronomy including flat lenses [183, 184] and tailored emissivity mm-wave radiators. These developments are interesting for the scientific program in this White Paper.

Focal plane arrays The polarimetric imager uses 3-color pixels with TES bolometers at frequencies below $450 \mathrm{GHz}$. The technology is extensively used on the ground over a narrower range of frequencies, and with higher optical loading. TES bolometers have been used aboard balloon instruments starting with the EBEX experiment in 2009 [185]. A flight of LiteBIRD will elevate the TRL of multi-color pixels with TES bolometers to spaceflight worthiness. The technical milestones for the filter-bank spectrometer are to: (1) demonstrate operation over a broader range of frequencies than have been used to date, which is $330-380 \mathrm{GHz}$ [179]; and (2) optimize the operation of the detectors to space loading. 
TES and KID readout technologies are progressing rapidly. By the 2030s we should expect multiplexing factors in the several thousands to have been thoroughly tested. Reduction in power consumption by FPGAs, ADCs, and DACs would further simplify the design of a 2030s survey.

\section{Conclusion}

Many questions in Cosmology remain unanswered. We do not know the nature of dark matter and dark energy, nor their modes of interaction, nor if extra light particles exist. We are unsure whether inflation did take place and what physics was at work in the very early Universe. We do not know the topology of the Universe, nor whether it is finite or infinite. We do not fully understand how structures form, and do not have an explanation for anomalies in the large-scale statistics of CMB anisotropies.

Those questions are tightly linked to fundamental physics, as we still have to reconcile the laws of gravitation with the standard model of particle interactions. They are also related to many domains in astrophysics, among which the role of baryons in structure formation, early stars and metals, and the formation of early galaxies.

Tiny signatures in the microwave sky emission encode the answers to those questions. With the development of relevant instrumentation, it is now becoming possible to envision a complete census of matter and radiation in the Hubble volume, through an appropriate program of observations in the microwave domain of the electromagnetic spectrum. At the level of accuracy we plan for, most of these observations can only be done from space.

The frequency range of the proposed survey concentrates more than $95 \%$ of the total radiative energy in the Universe. It is the most relevant to observe the high redshift Universe, offering a means to observe the evolution of the Universe over $99 \%$ of the cosmic time. This offers one of the best opportunities to investigate the interaction of matter and radiation all the way to redshift $2 \times 10^{6}$, as well as the distribution of matter in various forms and the growth of structure out to redshift $z \sim 8$, on all scales from that of the entire observable Universe down to individual galaxies.

Completing these observations requires an L-class space mission with spectropolarimetric instruments operating in the $10-2000 \mathrm{GHz}$ frequency range. As a strawman concept, we propose an observatory with three cryogenic instruments, two of them at the focus of a 3-m class telescope, for arcminute-scale multifrequency polarized imaging and moderate spectral resolution $(R=300)$ spectroscopy. A separate absolute spectrophotometer with degree-scale angular resolution will measure the absolute level of emission of the microwave sky in hundreds of frequency bands. Together, these three instruments will map with unprecedented accuracy the continuum emission, the line emission, and the integrated emission from all kinds of cosmological sources of radiation, all the way to the last scattering surface, and beyond.

The mission concept builds upon previous proposals and studies, with moderate extrapolations of demonstrated technology. We envision three modes of observations for a six-year mission: a full-sky survey for a broad picture of the Universe on a large 
scale; deep-patch surveys to map continuum and line emission back to the epoch of reionization; and observatory time open to the scientific community, for flexibility to address any other science question that may be relevant at the time of operation (see also Appendix A.4).

This proposed L-class mission offers a unique window on our Universe, with enormous science impact for cosmology, huge discovery potential, and high legacy value for many branches of astrophysics. We advocate that it should be part of the ESA science program for 2035-2050.

\section{Appendix A: Additional notes}

This appendix answers questions asked at the Voyage 2050 Workshop (29-31 October 2019, Madrid) by members of the Senior Committee and Topical Teams, and expands on science aspects that were only partially covered in the relevant submitted White Paper(s).

\section{A.1 Comparison of the proposed survey with existing and planned instruments}

The proposed spectro-polarimetric survey will map the full sky in microwaves with three instruments: a Polarized Imager and a Filterbank Spectrometer, both with arcminute-scale angular resolution, and an absolutely-calibrated Fourier Transform Spectrometer with degree-scale angular resolution.

The Polarized Imager is a successor to a series of CMB spaceborne instruments (COBE-DMR, WMAP, Planck), but is optimised for a larger set of science goals. This multi-purpose vision makes it stand out from other existing and planned microwave instruments specifically dedicated to the detection of inflationary gravitational waves. Even if the Polarized Imager onboard our proposed space mission is expected to reach the fundamental cosmic variance limit for all primary CMB science, the main design-driving science goal is to map dark matter, hot gas distribution and temperature, and the large-scale velocity flows over the entire sky, on all scales down to about 1 arcminute, up to redshift $z$ of a few. To that effect, the instrument has better sensitivity and more frequency bands than other wide-field CMB imagers, and better angular resolution than other space missions currently under study (10 times better than LiteBIRD, 2-3 times better than PICO). The resolution of $\sim 1$ arcmin or better at frequency above $300 \mathrm{GHz}$ will match the resolution of the future CMB-S4 groundbased multi-site observatory at lower frequency, allowing a combination of the data for an increase of the scientific reach of both experiments.

The Filterbank Spectrometer is an instrument with unique capabilities. Sensitive wide-field spectrometry over the full frequency range of 100-1000 GHz is impossible from the ground by reason of atmospheric absorption and emission. The instrument is designed for line intensity mapping (LIM) and high-redshift source detection using [C-II], [O-I], [O-II], [O-III], [N-II], and CO ladder lines up to $z \simeq 8$. The resulting hundreds of LIM maps will provide an unprecedented 3D view of the large-scale structure in the Universe at different stages of its evolution as traced by the combination of the different gas phases. These maps provide a direct way to 
observe the evolution of our Universe, from the time of formation of the first galaxies until today, for a clear understanding on how the evolution of galaxies connects to that of the intergalactic medium large-scale structure. The wide field of view, frequency span, angular resolution, spectral resolution, and sensitivity of this instrument make it possible to accurately separate the contribution from the different lines, and hence contributions at different redshifts, which is essential for cosmology. For direct source detection, spectroscopy with $R \simeq 300$ will overcome most of the confusion limits of broadband surveys. We anticipate the detection of tens of millions of star-forming galaxies between redshift 1 and 8 (including hundreds of sources in the Epoch of Reionization at $z>7$ ), and of more than one million proto-clusters of galaxies above redshift 2. For most of these sources, unambiguous redshift will be directly available thanks to the detection of more than one emission line. The detection of large samples of strongly lensed galaxies up to high redshifts makes it possible to trace, via high-resolution follow-up, the evolution of the internal structure and kinematics of star-forming galaxies on tens of parsec scales, which is the most direct way of learning about the complex physical processes in action in those highredshift objects. Finally, the detection of large samples of proto-clusters up to high $z$ allows us to reconstruct the growth history of the most massive present-day virialized halos well beyond what can be done with any other technique. More details about the science achievable with the high-redshift spectroscopic survey can be found in [45].

The Fourier Transform Spectrometer is designed to measure small departures of the CMB energy distribution from a perfect blackbody in the $10-2000 \mathrm{GHz}$ frequency range. This measurement opens new ways to learn about a wide range of earlyuniverse processes, in particular inflation and very high-energy particle interactions, and cannot be made from the ground. The FTS also complements higher angular resolution imaging and line spectroscopy achieved with the other two instruments, by measuring the total integrated emission (rather than only emission fluctuations across the sky). This offers a means to map the total microwave sky emission with absolute calibration and unprecedented angular and spectral resolution. For primary CMB science specifically, in combination with CMB imaging, the FTS will open novel ways to control systematics, reduce calibration uncertainties, and mitigate foregrounds to unprecedented precision, all building on and extending the long-standing legacy of COBE-FIRAS.

\section{A.2 Cost estimates and feasibility}

As the Voyage 2050 call did not ask for proposals for specific missions, but rather to argue for science themes, we did not estimate the cost of the preliminary mission profile outlined in the present White Paper. However, to ensure realism of the proposed survey, we constrained the concept on the basis of mild extrapolations of missions and instruments demonstrated to be feasible in other contexts. The most demanding aspects of the mission are the large cryogenic payload (3-meter-class telescope at $8 \mathrm{~K}$ ), and three instruments operating at sub-K temperature. A $3.5 \mathrm{~m}$ telescope has been launched on Herschel, but was passively cooled to $\sim 85 \mathrm{~K}$ rather than actively cooled to $8 \mathrm{~K}$ as proposed for our spectro-polarimetric survey. The feasibility of an $8 \mathrm{~K}$ mirror in the sub-mm has been demonstrated for SPICA for a $2.4 \mathrm{~m}$ mirror. Our 
proposed mission profile can build on these studies for a 3-meter class telescope at $8 \mathrm{~K}$.

The survey considers three instruments, which is not unusual for space observatories. The sub-K operating temperatures required for those instruments are demanding, but have been demonstrated on other missions (Herschel, Planck, Hitomi) and are being planned for Athena, SPICA, LiteBIRD. The cryogenic design can build on cooling chains developed for these missions. In the context of the PICO study in the US, the total cost of a polarized imager instrument matching our requirements was estimated to be about $\$ 160 \mathrm{M}$ for phases A-E (including the sub-K coolers). This cost estimate includes 30\% reserves for development (Phases $\mathrm{A}-\mathrm{D}$ ) and 13\% reserves for operations (Phase E). We anticipate similar costs for the other two instruments. Note that the survey would retain its high scientific interest even considering some down-scoping. The detailed trade-off between performance and cost will be subject to optimization at the time of a feasibility study. Many options of international partnership are open.

\section{A.3 Pursuing new physics in the microwaves}

The proposed survey will carry out new and transformative science from space. It is not an upgraded version of previous and successful CMB missions that would continue pursuing the same science objectives with better accuracy. Several of the observations described here will be realized for the first time, such as line intensity mapping across the whole sky, reconstruction of the 3D cosmic velocity field with CMB scattering and lensing, and opening many orders of magnitude of discovery space with the $\mathrm{CMB}$ spectral distortions. The proposed mission concept goes well beyond what can be envisaged from the upcoming ground-based CMB experiments. Accessing the high frequencies with multiple bands will be mandatory for unbiased component separation when the signals of interest lie orders of magnitude below Galactic and extragalactic foregrounds, and is out of reach of ground-based observatories.

\section{A.4 Synergy with other cosmological surveys}

In the context of the ESA consultation of the scientific community for the Voyage 2050 planning cycle, several White Papers advocate, in a way or another, for a census of the distribution of matter and energy in various forms across a large fraction of the observable Universe. Joint analyses involving stacking and cross correlation between different observables over large volumes of spacetime are very powerful, still emerging tools to probe in detail the physics at work across all of cosmic history.

Acknowledgements This work has received funding from the European Research Council (ERC) under the European Union's Horizon 2020 research and innovation program (grant agreement No 725456, CMBSPEC) as well as the Royal Society (grants UF130435 and RG140523). JARM acknowledges financial support from the Spanish Ministry of Science and Innovation (MICINN) under the project AYA201784185-P, and from the European Union's Horizon 2020 research and innovation programme under Grant Agreement 687312 (RADIOFOREGROUNDS). C.H.-M. acknowledges the support of the Spanish Ministry of Science through project PGC2018-097585-B-C21. Financial support from the ASI/Physics 
Department of the University of Roma-Tor Vergata agreement n. 2016-24-H.0 for study activities of the Italian cosmology community is acknowledged. JLB is supported by the Allan C. and Dorothy H. Davis Fellowship, and has been supported by the Spanish MINECO under grant BES-2015-071307, co-funded by the ESF during part of the development of this work.

Open Access This article is licensed under a Creative Commons Attribution 4.0 International License, which permits use, sharing, adaptation, distribution and reproduction in any medium or format, as long as you give appropriate credit to the original author(s) and the source, provide a link to the Creative Commons licence, and indicate if changes were made. The images or other third party material in this article are included in the article's Creative Commons licence, unless indicated otherwise in a credit line to the material. If material is not included in the article's Creative Commons licence and your intended use is not permitted by statutory regulation or exceeds the permitted use, you will need to obtain permission directly from the copyright holder. To view a copy of this licence, visit http://creativecommonshorg/licenses/by/4.0/.

\section{References}

1. Revéret, V., Sauvage, M., Adami, O., et al.: In: Lystrup, M., Perrin, M.D., Batalha, N., Siegler, N., Tong, E.C. (eds.) Space telescopes and instrumentation 2020: optical, infrared, and millimeter wave, vol. 11443, pp. 1082-1091. International Society for Optics and Photonics (SPIE) (2020). https://doi.org/10.1117/12.2562419

2. Planck Collaboration 2018 I: arXiv:1807.06205 (2018)

3. Riess, A.G., Casertano, S., Yuan, W., Macri, L.M., Scolnic, D.: ApJ 876(1), 85 (2019). https://doi. org/10.3847/1538-4357/ab1422

4. Bullock, J.S., Boylan-Kolchin, M.: ARA\&A 55(1), 343 (2017). https://doi.org/10.1146/annurevastro-091916-055313

5. Planck Collaboration 2018 VI: arXiv:1807.06209 (2018)

6. Planck Collaboration 2013 XVII: A\&A 571, A17 (2014). https://doi.org/10.1051/0004-6361/ 201321543

7. Sherwin, B.D., van Engelen, A., Sehgal, N., et al.: Phys. Rev. D 95(12), 123529 (2017). https://doi.org/10.1103/PhysRevD.95.123529

8. Simard, G., Omori, Y., Aylor, K., et al.: ApJ 860(2), 137 (2018). https://doi.org/10.3847/1538-4357/ aac264

9. Laureijs, R.: arXiv:0912.0914 (2009)

10. Yasini, S., Mirzatuny, N., Pierpaoli, E.: ApJL 873(2), L23 (2019). https://doi.org/10.3847/20418213/ab0bfe

11. Hotinli, S.C., Meyers, J., Dalal, N., Jaffe, A.H., Johnson, M.C., Mertens, J.B., Münchmeyer, M., Smith, K.M., van Engelen, A.: arXiv:1812.03167 (2018)

12. Staniszewski, Z., Ade, P.A.R., Aird, K.A., et al.: ApJ 701(1), 32 (2009). https://doi.org/10.1088/ 0004-637X/701/1/32

13. Hasselfield, M., Hilton, M., Marriage, T.A., et al.: JCAP 2013(7), 008 (2013). https://doi.org/10. 1088/1475-7516/2013/07/008

14. Bleem, L.E., Stalder, B., de Haan, T., et al.: ApJS 216(2), 27 (2015). https://doi.org/10.1088/0067$0049 / 216 / 2 / 27$

15. Planck Collaboration 2015 XXVII: A\&A 594, A27 (2016). https://doi.org/10.1051/0004-6361/ 201525823

16. Naab, T., Ostriker, J.P.: ARA\&A 55(1), 59 (2017). https://doi.org/10.1146/annurev-astro-081913040019

17. Basu, K., Erler, J., Chluba, J., et al.: BAAS 51(3), 302 (2019)

18. Remazeilles, M., Bolliet, B., Rotti, A., Chluba, J.: MNRAS 483, 3459 (2019). https://doi.org/10. 1093/mnras/sty3352

19. Remazeilles, M., Chluba, J.: arXiv:1907.00916 (2019)

20. Rephaeli, Y., Lahav, O.: ApJ 372, 21 (1991). https://doi.org/10.1086/169950

21. Hand, N., Addison, G.E., Aubourg, E., et al.: Phys. Rev. Lett. 109(4), 041101 (2012). https://doi.org/10.1103/PhysRevLett.109.041101

22. Planck Collaboration Int XIII: A\&A 561, A97 (2014). https://doi.org/10.1051/0004-6361/ 201321299 
23. Arnaud, M., Pratt, G.W., Piffaretti, R., Böhringer, H., Croston, J.H., Pointecouteau, E.: A\&A 517, A92 (2010). https://doi.org/10.1051/0004-6361/200913416

24. Challinor, A., Allison, R., Carron, J., et al.: JCAP 2018(4), 018 (2018). https://doi.org/10.1088/14757516/2018/04/018

25. Hanany, S., et al.: arXiv:1902.10541 (2019)

26. Dunkley, J., Calabrese, E., Sievers, J., et al.: JCAP 2013(7), 025 (2013). https://doi.org/10.1088/ 1475-7516/2013/07/025

27. Hu, W., Okamoto, T.: ApJ 574(2), 566 (2002). https://doi.org/10.1086/341110

28. Kravtsov, A.V., Borgani, S.: ARA\&A 50, 353 (2012). https://doi.org/10.1146/annurev-astro081811-125502

29. Overzier, R.A.: AAPR 24, 14 (2016). https://doi.org/10.1007/s00159-016-0100-3

30. Dannerbauer, H., et al.: A\&A 570, A55 (2014). https://doi.org/10.1051/0004-6361/201423771

31. Clements, D.L., et al.: MNRAS 461, 1719 (2016). https://doi.org/10.1093/mnras/stw1224

32. Casey, C.M., Narayanan, D., Cooray, A.: Phys. Rep. 541, 45 (2014). https://doi.org/10.1016/j. physrep.2014.02.009

33. Negrello, M., Gonzalez-Nuevo, J., De Zotti, G., Bonato, M., Cai, Z.Y., Clements, D., Danese, L., Dole, H., Greenslade, J., Lapi, A., Montier, L.: MNRAS 470, 2253 (2017). https://doi.org/10.1093/ mnras/stx 1367

34. Ivison, R.J., et al.: ApJ 772, 137 (2013). https://doi.org/10.1088/0004-637X/772/2/137

35. Wang, T., et al.: ApJ 828, 56 (2016). https://doi.org/10.3847/0004-637X/828/1/56

36. Miller, T.B., et al.: Nature 556, 469 (2018). https://doi.org/10.1038/s41586-018-0025-2

37. Oteo, I., et al.: ApJ 856, 72 (2018). https://doi.org/10.3847/1538-4357/aaa1f1

38. Dannerbauer, H., van Kampen, E., et al.: BAAS 51, 293 (2019)

39. Klaassen, P., Mroczkowski, T., Bryan, S., Groppi, C., Basu, K., Cicone, C., Dannerbauer, H., De Breuck, C., Fischer, W.J., Geach, J., Hatziminaoglou, E., Holland, W., Kawabe, R., Sehgal, N., Stanke, T., van Kampen, E.: arXiv e-prints (2019)

40. De Zotti, G., Bonato, M., Negrello, M., Trombetti, T., Burigana, C., Herranz, D., López-Caniego, M., Cai, Z.Y., Bonavera, L., González-Nuevo, J.: arXiv:1907.05323 (2019)

41. Cañameras, R., et al.: A\&A 581, A105 (2015). https://doi.org/10.1051/0004-6361/201425128

42. Harrington, K.C., et al.: MNRAS 458, 4383 (2016). https://doi.org/10.1093/mnras/stw614

43. Dannerbauer, H., Harrington, K., Díaz-Sánchez, A., Iglesias-Groth, S., Rebolo, R., Genova-Santos, R.T., Krips, M.: AJ 158, 34 (2019). https://doi.org/10.3847/1538-3881/aaf50b

44. Frye, B.L., et al.: ApJ 871, 51 (2019). https://doi.org/10.3847/1538-4357/aaeff7

45. Negrello, M., Bonato, M., Cai, Z.Y., Dannerbauer, H., De Zotti, G., Delabrouille, J., Scott, D.: PASA 37, e025 (2020). https://doi.org/10.1017/pasa.2020.16

46. Lagache, G., Puget, J.L., Dole, H.: ARA\&A 43, 727 (2005). https://doi.org/10.1146/annurev.astro. 43.072103.150606

47. Hill, R., Masui, K.W., Scott, D.: Appl. Spectrosc. 72(5), 663 (2018). https://doi.org/10.1177/ 0003702818767133

48. Odegard, N., Weiland, J.L., Fixsen, D.J., Chuss, D.T., Dwek, E., Kogut, A., Switzer, E.R.: Astrophys. J. 877(1), 40 (2019). https://doi.org/10.3847/1538-4357/ab14e8

49. Creque-Sarbinowski, C., Kamionkowski, M.: Phys. Rev. D98(6), 063524 (2018). https://doi.org/10. 1103/PhysRevD.98.063524

50. Maniyar, A., Béthermin, M., Lagache, G.: Astron. Astrophys. 614, A39 (2018). https://doi.org/10. 1051/0004-6361/201732499

51. Béthermin, M., Wang, L., Doré, O., Lagache, G., Sargent, M., Daddi, E., Cousin, M., Aussel, H.: Astron. Astrophys. 557, A66 (2013). https://doi.org/10.1051/0004-6361/201321688

52. Tucci, M., Desjacques, V., Kunz, M.: Mon. Not. Roy. Astron. Soc. 463(2), 2046 (2016). https://doi. org/10.1093/mnras/stw2086

53. Kovetz, E.D., et al.: arXiv e-prints (2017)

54. Sargent, M.T., Béthermin, M., Daddi, E., Elbaz, D.: ApJL 747(2), L31 (2012). https://doi.org/10. 1088/2041-8205/747/2/L31

55. Bonato, M., De Zotti, G., Leisawitz, D., Negrello, M., Massardi, M., Baronchelli, I., Cai, Z.Y., Bradford, C.M., Pope, A., Murphy, E.J., Armus, L., Cooray, A.: PASA 36, e017 (2019). https://doi.org/10.1017/pasa.2019.8

56. Schaye, J., Crain, R.A., Bower, R.G., et al.: MNRAS 446(1), 521 (2015). https://doi.org/10.1093/ mnras/stu2058 
57. Mashian, N., Sternberg, A., Loeb, A.: JCAP 2015(11), 028 (2015). https://doi.org/10.1088/1475$7516 / 2015 / 11 / 028$

58. Bernal, J.L., Breysse, P.C., Kovetz, E.D.: arXiv e-prints (2019)

59. Moradinezhad Dizgah, A., Keating, G.K.: Astrophys. J. 872(2), 126 (2019). https://doi.org/10.3847/ 1538-4357/aafd36

60. Bernal, J.L., Breysse, P.C., Gil-Marín, H., Kovetz, E.D.: arXiv e-prints (2019)

61. PRISM Collaboration, Andre, P., Baccigalupi, C., Barbosa, D., Bartlett, J., et al.: arXiv:1306.2259 (2013)

62. Hazumi, M., et al.: J. Low. Temp. Phys. 194(5-6), 443 (2019). https://doi.org/10.1007/s10909-01902150-5

63. Aguirre, J., et al.: JCAP 1902, 056 (2019). https://doi.org/10.1088/1475-7516/2019/02/056

64. Abazajian, K., Addison, G., Adshead, P., et al.: arXiv:1907.04473 (2019)

65. Ade, P.A.R., et al.: Phys. Rev. Lett. 121, 221301 (2018). https://doi.org/10.1103/PhysRevLett.121. 221301

66. Planck Collaboration 2018 X: arXiv e-prints (2018)

67. Ferrara, S., Kallosh, R.: Phys. Rev. D 94(12), 126015 (2016). https://doi.org/10.1103/PhysRevD.94. 126015

68. Kallosh, R., Linde, A., Wrase, T., Yamada, Y.: J. High Energy Phys. 2017(4), 144 (2017). https://doi.org/10.1007/JHEP04(2017)144

69. Planck Collaboration 2018 IX: arXiv e-prints (2019)

70. de Salas, P.F., Pastor, S.: JCAP 2016(7), 051 (2016). https://doi.org/10.1088/1475-7516/2016/07/051

71. Remazeilles, M., Banday, A.J., Baccigalupi, C., et al.: JCAP 2018(4), 023 (2018). https://doi.org/10.1088/1475-7516/2018/04/023

72. Delabrouille, J., de Bernardis, P., Bouchet, F.R., Achúcarro, A., Ade, P.A.R., Allison, R., Arroja, F., Artal, E., Ashdown, M., Baccigalupi, C., et al.: JCAP 2018(4), 014 (2018). https://doi.org/10.1088/1475-7516/2018/04/014

73. Planck Collaboration 2018 VII: arXiv:1906.02552 (2019)

74. Burigana, C., Carvalho, C.S., Trombetti, T., et al.: JCAP 2018(4), 021 (2018). https://doi.org/10.1088/1475-7516/2018/04/021

75. Zeldovich, Y.B., Sunyaev, R.A.: ApSS 4, 301 (1969). https://doi.org/10.1007/BF00661821

76. Sunyaev, R.A., Zeldovich, Y.B.: ApSS 7, 20 (1970). https://doi.org/10.1007/BF00653472

77. Burigana, C., Danese, L., de Zotti, G.: A\&A 246, 49 (1991)

78. Hu, W., Silk, J.: Phys. Rev. D 48, 485 (1993). https://doi.org/10.1103/PhysRevD.48.485

79. Chluba, J., Sunyaev, R.A.: MNRAS 419, 1294 (2012). https://doi.org/10.1111/j.1365-2966.2011. 19786.x

80. Khatri, R., Sunyaev, R.A.: JCAP 9, 016 (2012). https://doi.org/10.1088/1475-7516/2012/09/016

81. Chluba, J.: MNRAS 436, 2232 (2013). https://doi.org/10.1093/mnras/stt1733

82. Chluba, J., Jeong, D.: MNRAS 438, 2065 (2014). https://doi.org/10.1093/mnras/stt2327

83. Kogut, A., Fixsen, D.J., Chuss, D.T., Dotson, J., Dwek, E., Halpern, M., Hinshaw, G.F., Meyer, S.M., Moseley, S.H., Seiffert, M.D., Spergel, D.N., Wollack, E.J.: JCAP 7, 25 (2011). https://doi.org/10.1088/1475-7516/2011/07/025

84. PRISM Collaboration, André, P., Baccigalupi, C., Banday, A., Barbosa, D., et al.: JCAP 2014(2), 006 (2014). https://doi.org/10.1088/1475-7516/2014/02/006

85. Kogut, A., Abitbol, M.H., Chluba, J., Delabrouille, J., Fixsen, D., Hill, J.C., Patil, S.P., Rotti, A.: arXiv:1907.13195 (2019)

86. Sunyaev, R.A., Zeldovich, Y.B.: ApSS 9, 368 (1970). https://doi.org/10.1007/BF00649577

87. Daly, R.A.: ApJ 371, 14 (1991). https://doi.org/10.1086/169866

88. Chluba, J., Khatri, R., Sunyaev, R.A.: MNRAS 425, 1129 (2012). https://doi.org/10.1111/j.13652966.2012.21474.x

89. Chluba, J., Erickcek, A.L., Ben-Dayan, I.: ApJ 758, 76 (2012). https://doi.org/10.1088/0004-637X/ $758 / 2 / 76$

90. Chluba, J., et al.: Voyage 2050 Survey (2019)

91. Pajer, E., Zaldarriaga, M.: Phys. Rev. Lett. 109(2), 021302 (2012). https://doi.org/10.1103/ PhysRevLett.109.021302

92. Ganc, J., Komatsu, E.: Phys. Rev. D 86(2), 023518 (2012). https://doi.org/10.1103/PhysRevD.86. 023518

93. Remazeilles, M., Chluba, J.: MNRAS 478, 807 (2018). https://doi.org/10.1093/mnras/sty1034 
94. Cen, R., Ostriker, J.P.: ApJ 514, 1 (1999). https://doi.org/10.1086/306949

95. Refregier, A., Komatsu, E., Spergel, D.N., Pen, U.L.: Phys. Rev. D 61(12), 123001 (2000). https://doi.org/10.1103/PhysRevD.61.123001

96. Miniati, F., Ryu, D., Kang, H., Jones, T.W., Cen, R., Ostriker, J.P.: ApJ 542, 608 (2000). https://doi.org/10.1086/317027

97. Oh, S.P., Cooray, A., Kamionkowski, M.: MNRAS 342, L20 (2003). https://doi.org/10.1046/j.13658711.2003.06708.x

98. Hill, J.C., et al.: Phys. Rev. Lett. 115(26), 261301 (2015). https://doi.org/10.1103/PhysRevLett.115. 261301

99. Wright, E.L.: ApJ 232, 348 (1979). https://doi.org/10.1086/157294

100. Sazonov, S.Y., Sunyaev, R.A.: ApJ 508, 1 (1998). https://doi.org/10.1086/306406

101. Itoh, N., Kohyama, Y., Nozawa, S.: ApJ 502, 7 (1998). https://doi.org/10.1086/305876

102. Oh, S.P.: ApJ 527, 16 (1999). https://doi.org/10.1086/308077

103. Ponente, P.P., Diego, J.M., Sheth, R.K., Burigana, C., Knollmann, S.R., Ascasibar, Y.: MNRAS 410, 2353 (2011). https://doi.org/10.1111/j.1365-2966.2010.17611.x

104. Trombetti, T., Burigana, C.: MNRAS 437, 2507 (2014). https://doi.org/10.1093/mnras/stt2063

105. Seiffert, M., Fixsen, D.J., Kogut, A., Levin, S.M., Limon, M., Lubin, P.M., Mirel, P., Singal, J., Villela, T., Wollack, E., Wuensche, C.A.: ApJ 734, 6 (2011). https://doi.org/10.1088/0004-637X/ 734/1/6

106. Bowman, J.D., Rogers, A.E.E., Monsalve, R.A., Mozdzen, T.J., Mahesh, N.: Nature 555, 67 (2018). https://doi.org/10.1038/nature25792

107. Ahmed, Z., et al.: Science 327, 1619 (2010). https://doi.org/10.1126/science.1186112

108. Aprile, E., et al.: Phys. Rev. Lett. 109, 181301 (2012). https://doi.org/10.1103/PhysRevLett.109. 181301

109. Angloher, G., et al.: Eur. Phys. J. C76(1), 25 (2016). https://doi.org/10.1140/epjc/s10052-0163877-3

110. Agnese, R., et al.: Phys. Rev. Lett. 116(7), 071301 (2016). https://doi.org/10.1103/PhysRevLett.116. 071301

111. Tan, A., et al.: Phys. Rev. Lett. 117(12), 121303 (2016). https://doi.org/10.1103/PhysRevLett.117. 121303

112. Akerib, D.S., et al.: Phys. Rev. Lett. 118(2), 021303 (2017). https://doi.org/10.1103/PhysRevLett. 118.021303

113. Jungman, G., Kamionkowski, M., Griest, K.: Phys. Rep. 267, 195 (1996). https://doi.org/10.1016/ 0370-1573(95)00058-5

114. Feng, J.L., Rajaraman, A., Takayama, F.: Phys. Rev. Lett. 91(1), 011302 (2003). https://doi.org/10. 1103/PhysRevLett.91.011302

115. Feng, J.L., Rajaraman, A., Takayama, F.: Phys. Rev. D68(6), 063504 (2003). https://doi.org/10. 1103/PhysRevD.68.063504

116. Kusenko, A.: Phys. Rep. 481, 1 (2009). https://doi.org/10.1016/j.physrep.2009.07.004

117. Feng, J.L.: ARA\&A 48, 495 (2010). https://doi.org/10.1146/annurev-astro-082708-101659

118. Carr, B.J., Kohri, K., Sendouda, Y., Yokoyama, J.: Phys. Rev. D81(10), 104019 (2010). https://doi.org/10.1103/PhysRevD.81.104019

119. Marsh, D.J.E.: Phys. Rep. 643, 1 (2016). https://doi.org/10.1016/j.physrep.2016.06.005

120. Ellis, J., Gelmini, G.B., Lopez, J.L., Nanopoulos, D.V., Sarkar, S.: Nuclear Phys. B 373, 399 (1992). https://doi.org/10.1016/0550-3213(92)90438-H

121. Adams, J.A., Sarkar, S., Sciama, D.W.: MNRAS 301, 210 (1998). https://doi.org/10.1046/j.13658711.1998.02017.x

122. Chen, X., Kamionkowski, M.: Phys. Rev. D70(4), 043502 (2004). https://doi.org/10.1103/ PhysRevD.70.043502

123. Padmanabhan, N., Finkbeiner, D.P.: Phys. Rev. D72(2), 023508 (2005). https://doi.org/10.1103/ PhysRevD.72.023508

124. Galli, S., Iocco, F., Bertone, G., Melchiorri, A.: Phys. Rev. D 80(2), 023505 (2009). https://doi.org/10.1103/PhysRevD.80.023505

125. Slatyer, T.R., Padmanabhan, N., Finkbeiner, D.P.: Phys. Rev. D80(4), 043526 (2009). https://doi.org/10.1103/PhysRevD.80.043526

126. Slatyer, T.R., Wu, C.L.: Phys. Rev. D95(2), 023010 (2017). https://doi.org/10.1103/PhysRevD.95. 023010 
127. Poulin, V., Lesgourgues, J., Serpico, P.D.: JCAP 3, 043 (2017). https://doi.org/10.1088/1475-7516/ 2017/03/043

128. Wilkinson, R.J., Bœhm, C., Lesgourgues, J.: JCAP 2014, 011 (2014). https://doi.org/10.1088/14757516/2014/05/011

129. Dvorkin, C., Blum, K., Kamionkowski, M.: Phys. Rev. D89(2), 023519 (2014). https://doi.org/10. 1103/PhysRevD.89.023519

130. Wilkinson, R.J., Lesgourgues, J., Bœhm, C.: JCAP 2014, 026 (2014). https://doi.org/10.1088/14757516/2014/04/026

131. Gluscevic, V., Boddy, K.K.: Phys. Rev. Lett. 121, 081301 (2018). https://doi.org/10.1103/ PhysRevLett.121.081301

132. Kawasaki, M., Kohri, K., Moroi, T.: Phys. Rev. D71(8), 083502 (2005). https://doi.org/10.1103/ PhysRevD.71.083502

133. Jedamzik, K.: JCAP 3, 008 (2008). https://doi.org/10.1088/1475-7516/2008/03/008

134. Kawasaki, M., Kohri, K., Moroi, T., Takaesu, Y.: Phys. Rev. D97(2), 023502 (2018). https://doi.org/10.1103/PhysRevD.97.023502

135. Poulin, V., Serpico, P.D.: Phys. Rev. Lett. 114, 091101 (2015). https://doi.org/10.1103/PhysRevLett. 114.091101

136. Sarkar, S., Cooper, A.M.: Phys. Lett. B 148, 347 (1984). https://doi.org/10.1016/0370-2693(84) 90101-1

137. Ellis, J., Nanopoulos, D.V., Sarkar, S.: Nuclear Phys. B 259, 175 (1985). https://doi.org/10.1016/ 0550-3213(85)90306-2

138. Kawasaki, M., Sato, K.: Phys. Lett. B 169, 280 (1986). https://doi.org/10.1016/0370-2693(86) 90666-0

139. Hu, W., Silk, J.: Phys. Rev. Lett. 70, 2661 (1993). https://doi.org/10.1103/PhysRevLett.70.2661

140. Aalberts, J.L., Ando, S., Borg, W.M., Broeils, E., Broeils, J., Broeils, S., Kavanagh, B.J., Leguijt, G., Reemst, M., van Arneman, D.R., Vu, H.: Phys. Rev. D98(2), 023001 (2018). https://doi.org/10.1103/PhysRevD.98.023001

141. McDonald, P., Scherrer, R.J., Walker, T.P.: Phys. Rev. D63(2), 023001 (2001). https://doi.org/10.1103/PhysRevD.63.023001

142. Lyubarsky, Y.E., Sunyaev, R.A.: A\&A 123, 171 (1983)

143. Chluba, J., Sunyaev, R.A.: A\&A 501, 29 (2009). https://doi.org/10.1051/0004-6361/200809840

144. Chluba, J.: MNRAS 402, 1195 (2010). https://doi.org/10.1111/j.1365-2966.2009.15957.x

145. Chluba, J.: MNRAS 454, 4182 (2015). https://doi.org/10.1093/mnras/stv2243

146. Slatyer, T.R.: Phys. Rev. D93(2), 023521 (2016). https://doi.org/10.1103/PhysRevD.93.023521

147. Acharya, S.K., Khatri, R.: Phys. Rev. D 99(4), 043520 (2019). https://doi.org/10.1103/PhysRevD. 99.043520

148. Tashiro, H., Silk, J., Marsh, D.J.E.: Phys. Rev. D88(12), 125024 (2013). https://doi.org/10.1103/ PhysRevD.88.125024

149. Ejlli, D., Dolgov, A.D.: Phys. Rev. D90, 063514 (2014). https://doi.org/10.1103/PhysRevD.90. 063514

150. Mukherjee, S., Khatri, R., Wandelt, B.D.: JCAP 4, 045 (2018). https://doi.org/10.1088/1475-7516/ 2018/04/045

151. Dimastrogiovanni, E., Krauss, L.M., Chluba, J.: Phys. Rev. D94(2), 023518 (2016). https://doi.org/10.1103/PhysRevD.94.023518

152. Ostriker, J.P., Thompson, C.: ApJL 323, L97 (1987). https://doi.org/10.1086/185065

153. Tashiro, H., Sabancilar, E., Vachaspati, T.: JCAP 8, 035 (2013). https://doi.org/10.1088/1475-7516/ 2013/08/035

154. Ali-Haïmoud, Y., Chluba, J., Kamionkowski, M.: Phys. Rev. D115, 071304 (2015). https://doi.org/10.1103/PhysRevLett.115.071304

155. Diacoumis, J.A.D., Wong, Y.Y.Y.: JCAP 9, 011 (2017). https://doi.org/10.1088/1475-7516/2017/ 09/011

156. Kumar, S., Dimastrogiovanni, E., Starkman, G.D., Copi, C., Lynn, B.: Phys. Rev. D 99(2), 023521 (2019). https://doi.org/10.1103/PhysRevD.99.023521

157. Jedamzik, K., Katalinić, V., Olinto, A.V.: PRL 85, 700 (2000). https://doi.org/10.1103/PhysRevLett. 85.700

158. Sethi, S.K., Subramanian, K.: MNRAS 356, 778 (2005). https://doi.org/10.1111/j.1365-2966.2004. 08520.x 
159. Kunze, K.E., Komatsu, E.: JCAP 1, 009 (2014). https://doi.org/10.1088/1475-7516/2014/01/009

160. Wagstaff, J.M., Banerjee, R.: Phys. Rev. D92(12), 123004 (2015). https://doi.org/10.1103/ PhysRevD.92.123004

161. Dubrovich, V.K.: Sov. Astron. Lett. 1, 196 (1975)

162. Rybicki, G.B., dell'Antonio, I.P.: ApJ 427, 603 (1994). https://doi.org/10.1086/174170

163. Dubrovich, V.K., Stolyarov, V.A.: A\&A 302, 635 (1995)

164. Kholupenko, E.E., Ivanchik, A.V., Varshalovich, D.A.: Gravit. Cosmol. 11, 161 (2005)

165. Wong, W.Y., Seager, S., Scott, D.: MNRAS 367, 1666 (2006). https://doi.org/10.1111/j.1365-2966. 2006.10076.x

166. Rubiño-Martín, J.A., Chluba, J., Sunyaev, R.A.: MNRAS 371, 1939 (2006). https://doi.org/10.1111/ j.1365-2966.2006.10839.x

167. Chluba, J., Sunyaev, R.A.: A\&A 446, 39 (2006). https://doi.org/10.1051/0004-6361:20053988

168. Rubiño-Martín, J.A., Chluba, J., Sunyaev, R.A.: A\&A 485, 377 (2008). https://doi.org/10.1051/ 0004-6361:20078993

169. Ali-Haïmoud, Y.: Phys. Rev. D 87(2), 023526 (2013). https://doi.org/10.1103/PhysRevD.87.023526

170. Chluba, J., Sunyaev, R.A.: A\&A 478, L27 (2008). https://doi.org/10.1051/0004-6361:20078200

171. Sunyaev, R.A., Chluba, J.: Astron. Nachr. 330, 657 (2009). https://doi.org/10.1002/asna.200911237

172. Chluba, J., Ali-Haïmoud, Y.: MNRAS 456, 3494 (2016). https://doi.org/10.1093/mnras/stv2691

173. Shirron, P.J., Kimball, M.O., Fixsen, D.J., Kogut, A.J., Li, X., DiPirro, M.J.: Cryogenics 52, 140 (2012). https://doi.org/10.1016/j.cryogenics.2012.01.009. https://ui.adsabs.harvard.edu/\# abs/2012Cryo...52..140S

174. Shirron, P.J., Kimball, M.O., James, B.L., Muench, T., DiPirro, M.J., Bialas, T.G., Sneiderman, G.A., Porter, F.S., Kelley, R.L.: Cryogenics 74, 24 (2016). https://doi.org/10.1016/j.cryogenics.2015. 10.011

175. Brien, T.L.R., Castillo-Dominguez, E., Chase, S., Doyle, S.M.: J. Low Temp. Phys. 193(5), 805 (2018). https://doi.org/10.1007/s10909-018-2024-y

176. Suzuki, A., Arnold, K., Edwards, J., et al.: J. Low Temp. Phys. 176, 650 (2014). https://doi.org/10.1007/s10909-013-1049-5

177. Datta, R., Hubmayr, J., Munson, C., et al.: J. Low Temp. Phys. 176, 670 (2014). https://doi.org/10.1007/s10909-014-1134-4

178. Abitbol, M.H., Chluba, J., Hill, J.C., Johnson, B.R.: MNRAS 471, 1126 (2017). https://doi.org/10. 1093/mnras/stx1653

179. Endo, A., Karatsu, K., Tamura, Y., et al.: arXiv:1906.10216 (2019)

180. Baselmans, J.J.A., Bueno, J., Yates, S.J.C., Yurduseven, O., Llombart, N., Karatsu, K., Baryshev, A.M., Ferrari, L., Endo, A., Thoen, D.J., de Visser, P.J., Janssen, R.M.J., Murugesan, V., Driessen, E.F.C., Coiffard, G., Martin-Pintado, J., Hargrave, P., Griffin, M.: A\&A 601, A89 (2017). https://doi.org/10.1051/0004-6361/201629653

181. Bueno, J., Yurduseven, O., Yates, S.J.C., Llombart, N., Murugesan, V., Thoen, D.J., Baryshev, A.M., Neto, A., Baselmans, J.J.A.: Appl. Phys. Lett. 110(23), 233503 (2017). https://doi.org/10.1063/1.4985060

182. Origins STDT: Origins space telescope. https://asd.gsfc.nasa.gov/firs/ NASA (2019)

183. Aieta, F., Kats, M.A., Genevet, P., Capasso, F.: Science 347(6228), 1342 (2015). https://doi.org/10.1126/science.aaa2494. https://science.sciencemag.org/content/347/6228/1342

184. Pisano, G., Ng, M.W., Ozturk, F., Maffei, B., Haynes, V.: Appl. Opt. 52(11), 2218 (2013). https://doi.org/10.1364/AO.52.002218

185. EBEX Collaboration, Abitbol, M., Aboobaker, A.M., Ade, P., et al.: ApJS 239(1), 8 (2018). https://doi.org/10.3847/1538-4365/aae436

Publisher's note Springer Nature remains neutral with regard to jurisdictional claims in published maps and institutional affiliations. 


\section{Affiliations}

Jacques Delabrouille ${ }^{1,2}$ (D) . Maximilian H. Abitbol ${ }^{3}$ - Nabila Aghanim ${ }^{4}$. Yacine Ali-Haïmoud ${ }^{5}$. David Alonso ${ }^{3,6}$. Marcelo Alvarez ${ }^{7,8}$. Anthony J. Banday ${ }^{9}$. James G. Bartlett ${ }^{1,10}$. Jochem Baselmans ${ }^{11,12}$. Kaustuv Basu ${ }^{13}$. Nicholas Battaglia ${ }^{14}$. José Ramón Bermejo Climent ${ }^{15}$. José L. Bernal ${ }^{16}$. Matthieu Béthermin ${ }^{17}$. Boris Bolliet ${ }^{18} \cdot$ Matteo Bonato $^{19,20}$. François R. Bouchet ${ }^{21}$. Patrick C. Breysse ${ }^{22}$. Carlo Burigana ${ }^{19}$.

Zhen-Yi Cai ${ }^{23,24}$. Jens Chluba ${ }^{18}$. Eugene Churazov ${ }^{25,26}$.

Helmut Dannerbauer ${ }^{27}$. Paolo De Bernardis ${ }^{28,29}$. Gianfranco De Zotti ${ }^{20}$. Eleonora Di Valentino ${ }^{18}$. Emanuela Dimastrogiovanni ${ }^{30}$. Akira Endo ${ }^{11,31}$. Jens Erler $^{13}$ - Simone Ferraro 8,7 . Fabio Finelli ${ }^{15}$. Dale Fixsen ${ }^{32} \cdot$ Shaul Hanany $^{33}$. Luke Hart $^{18}$. Carlos Hernández-Monteagudo ${ }^{34}$. J. Colin Hill ${ }^{35,36}$.

Selim C. Hotinli ${ }^{37}$. Kenichi Karatsu ${ }^{11,12}$. Kirit Karkare ${ }^{38}$. Garrett K. Keating ${ }^{39}$. Ildar Khabibullin 25,26 . Alan Kogut ${ }^{40}$. Kazunori Kohri ${ }^{41,42}$. Ely D. Kovetz ${ }^{43}$. Guilaine Lagache $^{17}$. Julien Lesgourgues ${ }^{44}$. Mathew Madhavacheril ${ }^{45}$.

Bruno Maffei ${ }^{4}$. Nazzareno Mandolesi ${ }^{46,47}$. Carlos Martins ${ }^{48,49}$.

Silvia Masi ${ }^{28,29}$. John Mather ${ }^{40}$. Jean-Baptiste Melin ${ }^{2}$.

Azadeh Moradinezhad Dizgah ${ }^{50,51}$. Tony Mroczkowski ${ }^{52}$.

Suvodip Mukherjee ${ }^{21}$. Daisuke Nagai ${ }^{53}$. Mattia Negrello ${ }^{6}$.

Nathalie Palanque-Delabrouille ${ }^{2}$. Daniela Paoletti ${ }^{15}$. Subodh P. Patil ${ }^{54}$.

Francesco Piacentini ${ }^{28,29}$. Srinivasan Raghunathan ${ }^{55}$. Andrea Ravenni ${ }^{18}$. Mathieu Remazeilles ${ }^{18}$. Vincent Revéret ${ }^{2}$. Louis Rodriguez ${ }^{2}$. Aditya Rotti ${ }^{18}$. Jose-Alberto Rubiño Martin 27,56 . Jack Sayers ${ }^{57}$. Douglas Scott ${ }^{58}$.

Joseph Silk $21,59,60$. Marta Silva ${ }^{61}$. Tarun Souradeep ${ }^{62} \cdot$ Naonori Sugiyama $^{63}$. Rashid Sunyaev 25,26,35 • Eric R. Switzer ${ }^{40}$. Andrea Tartari ${ }^{64}$.

Tiziana Trombetti ${ }^{19}$ • Íñigo Zubeldia 65,66 
1 Laboratoire Astroparticule et Cosmologie (APC), CNRS/IN2P3, Université Paris Diderot, 75205 Paris Cedex 13, France

2 IRFU, CEA, Université Paris Saclay, 91191 Gif-sur-Yvette, France

3 University of Oxford, Denys Wilkinson Building, Keble Road, Oxford, OX1 3RH, UK

4 Institut d'Astrophysique Spatiale (IAS), CNRS (UMR8617), Université Paris-Sud, Bâtiment 121, 91405 Orsay, France

5 Center for Cosmology and Particle Physics, Department of Physics, New York University, New York, NY 10003, USA

6 School of Physics and Astronomy, Cardiff University, The Parade, Cardiff, CF24 3AA, UK

7 Berkeley Center for Cosmological Physics, University of California, Berkeley, CA 94720, USA

8 Lawrence Berkeley National Laboratory, One Cyclotron Road, Berkeley, CA 94720, USA

9 IRAP, 9, av du Colonel Roche, BP 44346, 31028 Toulouse Cedex 4, France

10 Jet Propulsion Laboratory, California Institute of Technology, 4800 Oak Grove Drive, Pasadena, CA 91109, USA

11 Faculty of Electrical Engineering, Mathematics and Computer Science, Delft University of Technology, Mekelweg 4, 2628 CD Delft, The Netherlands

12 SRON, Netherlands Institute for Space Research, Sorbonnelaan 2, 3584 CA Utrecht, The Netherlands

13 Argelander-Institut für Astronomie, Universität Bonn, Auf dem Hügel 71, 53121 Bonn, Germany

14 Department of Astronomy, Cornell University, Ithaca, NY 14853, USA

15 INAF - Osservatorio di Astrofisica e Scienza dello Spazio, Via Gobetti 101, 40129 Bologna, Italy

16 ICC, University of Barcelona, IEEC-UB, Martí i Franques 1, E08028 Barcelona, Spain

17 Aix Marseille Université, CNRS, CNES, LAM, Marseille, France

18 JBCA, Department of Physics \& Astronomy, The University of Manchester, Manchester M13 9PL, $\mathrm{UK}$

19 INAF - Istituto di Radioastronomia, Via Piero Gobetti 101, 40129, Bologna, Italy

20 INAF - Osservatorio Astronomico di Padova, Vicolo Osservatorio 5, 35122, Padova, Italy

21 Institut d'Astrophysique de Paris, 98 bis Boulevard Arago, 75014 Paris, France

22 Canadian Institute for Theoretical Astrophysics, University of Toronto, Ontario, M5S 3H8, Canada

23 CAS Key Laboratory for Research in Galaxies and Cosmology, Department of Astronomy, USTC, Hefei, Anhui 230026, China

24 School of Astronomy and Space Science, USTC, Hefei 230026, China

25 Max-Planck-Institut für Astrophysik, Karl-Schwarzschild Str. 1, 85741 Garching, Germany

26 Space Research Institute (IKI), Profsoyuznaya 84/32, Moscow 117997, Russia

27 Instituto de Astrofísica de Canarias, C/ Vía Láctea 39020 La Laguna, Tenerife, Spain Physics Department, Sapienza University of Rome, Piazzale Aldo Moro 5, 00185, Rome, Italy INFN sezione di Roma, P.le A. Moro 2, 00815 Roma, Italy

School of Physics, The University of New South Wales, Sydney, NSW 2052, Australia

Kavli Institute of Nanoscience, Faculty of Applied Sciences, Delft University of Technology, Lorentzweg 1, 2628 CJ Delft, The Netherlands

Department of Astronomy, University of Maryland, College Park, MD 20742-2421, USA

School of Physics and Astronomy, University of Minnesota/Twin Cities, Minneapolis, MN 55455, USA

Centro de Estudios de Física del Cosmos de Aragón (CEFCA), Plaza San Juan, 1, planta 2, E-44001, Teruel, Spain

Institute for Advanced Study, Princeton, NJ 08540, USA

Center for Computational Astrophysics, Flatiron Institute, 162 5th Avenue, New York, NY 10010, USA

Blackett Laboratory, Imperial College London, Prince Consort Road, London SW7 2AZ, UK 
38

Kavli Institute for Cosmological Physics, University of Chicago, Chicago, IL 60637, USA

Harvard-Smithsonian Center for Astrophysics, 60 Garden Street, Cambridge, MA 02138, USA

NASA/GSFC, Mail Code: 665, Greenbelt, MD 20771, USA

KEK and Sokendai, Tsukuba 305-0801, Japan

Kavli IPMU, University of Tokyo, Kashiwa 277-8582, Japan

Department of Physics, Ben-Gurion University, Be'er Sheva 84105, Israel

Institute for Theoretical Particle Physics and Cosmology (TTK), RWTH Aachen University, 52056 Aachen, Germany

Perimeter Institute for Theoretical Physics, Waterloo, ON N2L 2Y5, Canada

Dipartimento di Fisica e Scienze della Terra, Universit'a degli Studi di Ferrara, Via Saragat 1, 44100 Ferrara, Italy

INAF - Osservatorio di Astrofisica e Scienza dello Spazio di Bologna, via Gobetti 101, 40129 Bologna, Italy

Centro de Astrofísica da Universidade do Porto, Rua das Estrelas, 4150-762 Porto, Portugal Instituto de Astrofísica e Ciências do Espaço, CAUP, Rua das Estrelas, 4150-762 Porto, Portugal

Department of Theoretical Physics and Centre for Astroparticle Physics, University of Geneva, $\mathrm{CH}-$ 1211 Geneva, Switzerland

Department of Physics, Harvard University, 17 Oxford St., Cambridge, MA 02138, USA

European Southern Observatory, Karl-Schwarzschild-Strasse 2, Garching 85748, Germany

Department of Physics \& Department of Astronomy, Yale University, New Haven, CT 06520, USA

Niels Bohr International Academy and Discovery Center, Blegdamsvej 17, 2100 Copenhagen, Denmark

Department of Physics and Astronomy, University of California, Los Angeles, CA 90095, USA

Departamento de Astrofísica, Universidad de La Laguna, 38206 La Laguna, Tenerife, Spain

California Institute of Technology, 1200 E. California Boulevard, MC 367-17, Pasadena, CA 91125 , USA

Department of Physics and Astronomy, University of British Columbia, 6224 Agricultural Road, Vancouver, V6T 1Z1, Canada

Department of Physics and Astronomy, The Johns Hopkins University, 3701 San Martin Drive, Baltimore, MD 21218, USA

Department of Physics and Beecroft Institute for PAC, University of Oxford, 1 Keble Road, Oxford, OX1 3RH, UK

Institute of Theoretical Astrophysics, University of Oslo, P.O.B 1029 Blindern, 0315 Oslo, Norway

Indian Institute of Science Education and Research (IISER), Dr. Homi Bhabha Road, Pashan, Pune 411008, India

National Astronomical Observatory of Japan, Mitaka, Tokyo 181-8588, Japan

INFN Sezione di Pisa, Largo B. Pontecorvo 3, 56127 Pisa, Italy

Institute of Astronomy, Madingley Road, Cambridge CB3 OHA, UK

Kavli Institute for Cosmology Cambridge, Madingley Road, Cambridge CB3 OHA, UK 